\title{
Some Remarkable Degenerations of Quantum Groups
}

\author{
C. De Concini ${ }^{1}$, V. G. Kac ${ }^{2, \star}$, and C. Procesi ${ }^{3}$ \\ 1 Scuola Normale Superiore, Pisa, Italy \\ 2 Department of Mathematics, MIT, Cambridge, MA 02139, USA \\ 3 Dipartimento di Matematica, Universit degli studi di Roma "La Sapienza", Rome, Italy
}

Received November 12, 1992; in revised form April 2, 1993

\section{To Armand Borel on his $70^{\text {th }}$ birthday}

\begin{abstract}
We show that an irreducible representation of a quantized enveloping algebra $U_{\varepsilon}$ at a $\ell^{\text {th }}$ root of 1 has maximal dimension $\left(=\ell^{N}\right)$ if the corresponding symplectic leaf has maximal dimension $(=2 N)$. The method of the proof consists of a construction of a sequence of degenerations of $U_{\varepsilon}$, the last one being a $q$ commutative algebra $U_{\varepsilon}^{(2 N)}$. This allows us to reduce many problems concerning $U_{\varepsilon}$ to that concerning $U_{\varepsilon}^{(2 N)}$.
\end{abstract}

\section{Introduction}

In the papers [DC-K, DC-K-P] the quantized enveloping algebras introduced by Drinfeld and Jimbo have been studied in the case $q=\varepsilon$, a primitive $l^{\text {th }}$ root of 1 with $l$ odd (cf. Sect. 4 for the basic definitions and relevant theorems). Let us recall for the moment only that such algebras are canonically constructed starting from a symmetrizable Cartan matrix of finite type and in particular we can talk of the associated classical objects (the root system, the simply connected algebraic group $G$, etc.). For such an algebra the irreducible representations have dimension bounded by $d:=l^{N}$, where $N$ is the number of positive roots, and the set of irreducible representations has a canonical map, called the restricted central character, to the big cell of the group $G$. In the same papers it has been shown in a precise sense that the representations look alike over points lying in the same conjugacy classes, and thus it is natural to analyze the structure of the representations associated to a given conjugacy class. This seems to be a rather difficult task. It is clear, however, that the structure of an irreducible representation $V$ is closely related to the geometry of the corresponding conjugacy class $\mathscr{Q}_{V}$. In particular, we conjectured in [DC-K-P] that $\operatorname{dim} V$ is always divisible by $\ell^{\frac{1}{2} \operatorname{dim} \mathscr{C}_{V}}$ (cf. [W-K]).

\footnotetext{
* Supported in part by the NSF grant DMS-9103792
} 
In this paper we succeed in treating the case of regular classes, i.e. conjugacy classes of maximal dimension. For these classes we prove our main theorem which we reformulate here as follows:

Theorem 5.1. The irreducible representations having as restricted central character a regular element have maximal dimension $d$.

The method used to prove this theorem may have an independent interest and consists of a degeneration argument (Lemma 1.5). In practice we degenerate our noncommutative algebra to a much simpler algebra (a twisted polynomial algebra) for which the representation theory is very simple. We prove that the representations we are analyzing degenerate in a suitable sense to representations of maximal dimension $d$, then an obvious semicontinuity of the dimension finishes the proof.

There are some interesting features in this degeneration argument which are connected to the combinatorics of the root system and the structure of the central characters of the fundamental representations of the algebraic group $G$, considered as functions on the big cell. These topics are explained in Sects. 3 and 4.

The structure of the paper is the following. In Sects. 1 and 2 we develop the necessary noncommutative algebra, i.e. a general theory of filtered algebras, their associated Rees and graded algebras and representations of twisted polynomial rings. Some of these results are well known but not easily traceable in the literature. In Sect. 3 we discuss the combinatorial aspects of root systems which are necessary for the study of the twisted polynomial algebra to which the quantum group degenerates. In Sect. 4 we recall the theory of quantum groups necessary for this paper, and finally in Sect. 5 we formulate the main theorems and give their proofs.

The variety of representations of the quantum group $U_{\varepsilon}$ provides a large set of solutions to the Yang-Baxter equation. These solutions have been already used in the study of the generalized chiral Potts models. We feel that our degeneration argument may help in obtaining explicit construction of representations of $U_{\varepsilon}$ and thereby finding new solutions to the Yang-Baxter equations on the one hand, and may clarify the structure of relevant statistical models on the other hand. We are grateful to the referee for suggesting to add comments about relevance to problems in physics.

\section{Finite Dimensional Representations of Algebras and Filtrations}

1.1. In this section we will collect some well known definitions and properties of finite dimensional representations of algebras. Let $A$ be an associative algebra with a unit element 1 over a field $\mathbb{F}$ and let us denote by $\overline{\mathbb{F}}$ the algebraic closure of $\mathbb{F}$. For an algebra $A$ we denote by $\operatorname{Spec} A$ the set of all equivalence classes of finite dimensional irreducible representations over $\overline{\mathbb{F}}$ so that, if $A$ is a finitely generated commutative algebra over $\mathbb{\mathbb { F }}$ we are in fact thinking of the maximal spectrum. If $Z$ is the center of $A$, then (by Schur's lemma) we have a canonical map (the central character map)

$$
\operatorname{Spec} A \stackrel{\chi}{\rightarrow} \operatorname{Spec} Z \text {. }
$$

A good theory of finite dimensional representations can be developed when the algebra $A$ is finitely generated over $\mathbb{F}$, is a finite module over its center $Z$ (this already implies that every irreducible module is finite dimensional) and has a suitable trace map (cf. [A] and [P1-2-3-4]). Let us first consider $A$ to be an order in a finite dimensional central simple algebra $D$. This means that the center $Z$ is a domain, $A$ is torsion free over $Z$ and, we have $D=A \otimes_{Z} Q(Z)$, where $Q(Z)$ is the field 
of fractions of $Z$. $A$ embeds naturally in $D$ which is its ring of fractions. If $\overline{Q(Z)}$ denotes the algebraic closure of $Q(Z)$ we have that $A \otimes_{Z} \overline{Q(Z)}$ is the full ring $M_{d}(\overline{Q(Z)})$ of $d \times d$ matrices over $\overline{Q(Z)}$. Hence we have on $D$ (and on $A$ ) the usual trace map $\operatorname{tr}: D \rightarrow Q(Z)$. It is well-known that $\operatorname{tr}(A) \subset Z$ if $A$ is a maximal order [P1].

Definition 1.1. (a) The number $d$ is called the degree of $A$.

(b) A d-dimensional representation of $A$ is a homomorphism $\varrho: A \rightarrow M_{d}(\overline{\mathbb{F}})$ compatible with the trace map.

Given a finitely generated algebra $A=\mathbb{F}\left[a_{1}, a_{2}, \ldots, a_{n}\right]$ and an integer $d$, a $d$ dimensional representation with coefficients in $\overline{\mathbb{F}}$ is a homomorphism of $A$ to $M_{d}(\overline{\mathbb{F}})$ and hence it is given by an $n$-tuple of $d \times d$ matrices satisfying some algebraic equations. Hence the set of such representations is an affine algebraic subvariety of the space of $n$-tuples of matrices, closed under the action by conjugation by the general linear group. It is known cf. ([A]) that the closed orbits of this action are the semisimple representations, and that given any representation the associated semisimple representation lies in the closure of its orbit. Thus one can apply invariant theory to this setting. In particular if we are in characteristic 0 we have that all invariants of $n$-tuples of matrices are generated by traces of monomials. This suggests ([P4]) to introduce a category of algebras with trace, of which maximal orders are a special case. The idea is that once we have a trace map in an algebra in characteristic 0 we can define formally the elementary symmetric functions in the eigenvalues of any element $a$ by declaring that $\operatorname{tr}\left(a^{k}\right)$ should be the sum of the $k^{t h}$ powers and using the formal identities between elementary symmetric functions and power sums. Then we can formally define for every integer $d$ a characteristic polynomial $\chi_{d, a}{ }^{[t]}$ for any element $a$ in $A$. This formal polynomial is useful in representation theory if we have the formal Cayley-Hamilton theorem, that is if $\chi_{d, a}[a]=0$ for every $a$. In this case we have a canonical commutative algebra $B$ with an action of the group $P G L(d, \mathbb{F})$ and an isomorphism of $A$ with the subring of $M_{d}(B)=M_{d}(\mathbb{F}) \otimes B$ of invariants under the diagonal action of $P G L(d, \mathbb{F})$. The ring of invariants of $B$ under the action of $P G L(d, \mathbb{F})$ equals the image $C$ in $A$ of the trace map, its spectrum can be identified with the equivalence classes of semisimple representations of $A$ of dimension $d$ and compatible with the trace map.

Some consequences of this picture are the following. Suppose $A$ is as before. Fixing any positive integer $k$ we can define a new $\operatorname{trace} \operatorname{tr}_{k}(a):=k \operatorname{tr}(a)$, and it is easy to see that, if $A$ satisfies the $d^{t h}$ characteristic polynomial under the original trace, it does satisfy the $k d^{t h}$ characteristic polynomial under the new trace. Then the same algebra $C$ parametrizes equivalence classes of $k d$-dimensional semisimple representations and it is easy to see that these are just obtained from the previous $d$-dimensional representations by considering each such representation with multiplicity $k$.

A second consequence is following. Suppose that $Z \subset C$ is a subring and that $C$ is a finite extension of $Z$ of degree $h$. Consider the reduced trace $\operatorname{tr}_{C / Z}: C \rightarrow Z$ and the composition $\operatorname{tr}_{A / Z}(a):=\operatorname{tr}_{C / Z}(\operatorname{tr}(a))$. Then under the same hypotheses as before the algebra $A$ equipped with this new trace satisfies the $h d^{t h}$ characteristic polynomial and $\operatorname{Spec} Z$ parametrizes equivalence classes of $h d$-dimensional representations. The picture is the following. Call $\pi: \operatorname{Spec} C \rightarrow \operatorname{Spec} Z$ the finite map of spectra. Given a point $P \in \operatorname{Spec} Z$ one defines $\pi^{-1}(P)=\sum h_{i} P_{i}$ as a positive 0 cycle. Each $P_{i}$ corresponds to a semisimple representation $\rho_{i}$ of $A$ of dimension $d$ and thus $P$ corresponds to $\sum h_{i} p_{i}$. 
We shall assume that $A$ is a finitely generated algebra over a field $\mathbb{F}$ and that it an order closed under trace and let $Z$ be its center. Then using the above arguments one obtains [A, P1]:

Theorem 1.1. (a) $Z$ is finitely generated and $A$ is a finite module over $Z$.

(b) The points of Spec $Z$ parametrize equivalence classes of d-dimensional semisimple representations.

(c) The canonical map $\operatorname{Spec} A \stackrel{\chi}{\rightarrow} \operatorname{Spec} Z$ is surjective and each fiber consists of all those irreducible representations of $A$ which are irreducible components of the corresponding semisimple representation. In particular each irreducible representation of $A$ has dimension at most $d$.

(d) The set

$$
\Omega_{A}=\{a \in \operatorname{Spec} Z \mid \text { the corresponding semisimple representation is irreducible }\}
$$
is a non-empty Zariski open set.

If $Z$ is finitely generated module over a subalgebra $Z_{0}$, we can consider the finite surjective morphism

$$
\operatorname{Spec} Z \stackrel{\tau}{\rightarrow} \operatorname{Spec} Z_{0} \text {. }
$$

Then by the properness of $\tau$ we get that the set $\Omega_{A}^{0}:=\left\{a \in \operatorname{Spec} Z_{0} \mid \tau^{-1}(a) \subset \Omega_{A}\right\}$ is a Zariski dense open subset of $\operatorname{Spec} Z_{0}$.

For a given algebra $A$ the problem of the study of its spectrum can be thus naturally divided in two steps. First one has to develop a geometric description of $\operatorname{Spec} Z$, then for each point of Spec $Z$ we need a description of the corresponding semisimple representations, i.e. of its irreducible components and multiplicities. In Sect. 5 we will discuss this problem in the case of quantum groups using a degeneration method based on suitable filtrations. In the rest of Sect. 1 we shall develop the necessary formalism.

1.2. An algebra $A$ is called $\left(\mathbb{Z}_{+}^{-}\right)$filtered if $A=\bigcup_{j \in \mathbb{Z}_{+}} A_{j}$ is a union of $\mathbb{F}$-submodules $A_{j}$ such that the following two properties hold:

$$
\begin{gathered}
1 \in A_{0} \subset A_{1} \subset A_{2} \subset \ldots, \\
A_{i} A_{j} \subset A_{i+j} .
\end{gathered}
$$

Let $\bar{A}=\bigoplus_{j \in \mathbb{Z}_{+}}\left(A_{j} / A_{j-1}\right)$ be the associated graded algebra. Given $a \in A$, we let $\operatorname{deg} a$ be the minimal $j$ for which $a \in A_{j}$, and let $\bar{a}$ be the image of $a$ in $A_{j} / A_{j-1}$. For a subset $S$ of $A$ we let $\bar{S}=\{\bar{a} \in \bar{A}$ where $a \in S\}$. For an ideal $I$ we will, by abuse of notations, indicate by $\bar{I}$ not just the previously defined set of homogeneous elements but also their (direct) sum.

Lemma 1.2. Let $A$ be a filtered algebra.

(a) If $a, b \in A$ and $\bar{a} \bar{b} \neq 0$, then $\overline{a b}=\bar{a} \bar{b}$; in particular if $\bar{A}$ has no zero divisors then $A$ has no zero divisors.

(b) Let $B$ be a subalgebra of $A$ with induced filtration. Let $\underline{a}_{1}, \underline{a}_{2}, \ldots$ be homogeneous generators of the left $\bar{B}$-module $\bar{A}$. Let $a_{1}, a_{2}, \ldots \in A$ be such that $\bar{a}_{1}=\underline{a}_{1}, \bar{a}_{2}=$ $\underline{a}_{2} \ldots$ Then any element $a$ of $A$ can be written in the form

$$
a=\sum_{i} b_{i} a_{i}, \quad \text { where } \quad \operatorname{deg} a_{i}+\operatorname{deg} b_{i} \leq \operatorname{deg} a .
$$


Proof. (a) is standard. In order to prove (b) note that we may write $\bar{a}=\sum_{i} \underline{b}_{i} \underline{a}_{i}$, where $\underline{b}_{i}$ are some homogeneous elements of $\bar{B}$ such that $\operatorname{deg} \underline{a}_{i}+\operatorname{deg} \underline{b}_{i}=\operatorname{deg} \bar{a}$. Taking $b_{i} \in B$ such that $\bar{b}_{\imath}=\underline{b}_{\imath}$, we obtain $a=\sum_{i} b_{i} a_{i}+a^{\prime}$, where $\operatorname{deg} a^{\prime}<\operatorname{deg} a$, and we apply the inductive assumption to $a^{\prime}$ (in degree 0 it is clear).

1.3. Let $A$ be a filtered algebra and let $A[t]$ (resp. $A\left[t, t^{-1}\right]$ ) denote the ring of polynomials (resp. Laurent polynomials) over $A$. The Rees algebra $\mathscr{R}(A)$ of $A$ is the following subalgebra of $A[t]$ :

$$
\mathscr{B}(A)=\sum_{j \in \mathbb{Z}_{+}} A_{j} t^{j} .
$$

The following properties of the algebra $\mathscr{R}(A)$ are obvious:

Lemma 1.3. (a) If $A$ has no zero divisors, then the same is true for $\mathscr{R}(A)$.

(b) If $\bar{A}$ is generated by homogeneous elements $\underline{a}_{1}, \underline{a}_{2}, \ldots$ of degree $r_{1}, r_{2}, \ldots$, then $\mathscr{R}(A)$ is generated by the elements $t, t^{r_{1}} a_{1}, t^{r_{2}} a_{2}, \ldots$, where the $a_{i}$ lift the $\underline{a}_{i}$.

(c) $A\left[t, t^{-1}\right]=\mathscr{B}(A)\left[t^{-1}\right]$.

(d) $\mathscr{R}(A) /(t) \simeq \bar{A}$.

(e) $B \subset A$ is a subalgebra with induced filtration, then $\mathscr{R}(B) \subset \mathscr{B}(A)$.

Remark 1.3. It follows from Lemma $1.3 \mathrm{c}$ that

$$
\text { degree } \bar{A} \leq \text { degree } \mathscr{R}(A) \text {. }
$$

From part (d) of the same lemma we deduce

$$
\text { degree } \bar{A} \leq \text { degree } A \text {. }
$$

The following proposition follows from Lemma 1.2b.

Proposition 1.3. Let $A$ be a filtered algebra, and let $B$ be a subalgebra of $A$. Let $a_{1}, a_{2}, \ldots$ be elements of $A$ of degrees $r_{1}, r_{2}, \ldots$ such that $\bar{A}$ is a left $\bar{B}$ module on generators $\bar{a}_{1}, \bar{a}_{2}, \ldots$ Then $\mathscr{R}(A)$ is a left $\mathscr{B}(B)$-module on generators $t^{r_{1}} a_{1}, t^{r_{2}} a_{2}, \ldots$

1.4. Lemma 1.4. Let $A$ be a filtered algebra and $I$ its ideal, then $\bar{I}$ is an ideal in $\bar{A}$, and if $H$ is the ideal of $A\left[t, t^{-1}\right]$ generated by $I$, then in $\bar{A}$ we have:

$$
(H \cap \mathscr{R}(A)+t \mathscr{R}(A)) / t \mathscr{B}(A)=\bar{I} .
$$

Proof. Clear.

In general it is difficult to determine generators for $\bar{I}$, therefore the next proposition is particularly useful when it can be applied.

Proposition 1.4. Let $A$ be a commutative filtered algebra and let $a_{1}, \ldots, a_{n} \in A$ be such that $\bar{a}_{1}, \ldots, \bar{a}_{n}$ is a regular sequence of $\bar{A}$. Let $I=\left(a_{1}, \ldots, a_{n}\right)$ be the ideal of A generated by $a_{1}, \ldots, a_{n}$. Then

(a) $a_{1}, \ldots, a_{n}$ is a regular sequence in $A$.

(b) The ideal $\bar{I}$ of $\bar{A}$ is generated by the elements $\bar{a}_{1}, \ldots, \bar{a}_{n}$.

Proof. (a) Suppose that $a_{1}, \ldots, a_{n}$ is not a regular sequence of $A$. Then there exist a $k \leq n$ and $b_{1}, \ldots, b_{k} \in A$ such that

$$
\sum_{j=1}^{k} a_{j} b_{\jmath}=0 \quad \text { and } \quad b_{k} \notin\left(a_{1}, \ldots, a_{k-1}\right) \text {. }
$$


We may assume that $d:=\max _{i} \operatorname{deg} a_{\imath} b_{\imath}$ is minimal possible for all such relations and (reordering if necessary) that for some $m \geq 1$ :

$$
d=\operatorname{deg} a_{1} b_{1}=\ldots=\operatorname{deg} a_{m} b_{m} \text { and } d>\operatorname{deg} a_{j} b_{j} \text { for } j>m .
$$

Then

$$
\sum_{i=1}^{m} \bar{a}_{i} \bar{b}_{i}=0
$$

This reorders the $a_{\imath}$, but since $\bar{A}$ is graded and the elements $\bar{a}_{1}, \ldots, \bar{a}_{n}$ are homogeneous we have that $\bar{a}_{1}, \ldots, \bar{a}_{m}$ is a regular sequence in $\bar{A}$ and the corresponding first Koszul homology group $H_{1}\left(\bar{A} ; \bar{a}_{1} ; \ldots, \bar{a}_{m}\right)$ vanishes [B]. Hence (1.4.3) implies that there exists a skew-symmetric $m \times m$ matrix $\bar{B}$ with homogeneous entries over $\bar{A}$ such that

$$
\left(\bar{b}_{1}, \ldots, \bar{b}_{m}\right)=\left(\bar{a}_{1}, \ldots, \bar{a}_{m}\right) \bar{B} .
$$

Let $B$ be a skew-symmetric matrix over $A$ whose image in $\bar{A}$ is $\bar{B}$. Let

$$
\left(b_{1}^{\prime}, \ldots, b_{m}^{\prime}\right)=\left(a_{1}, \ldots, a_{m}\right) B .
$$

Then $\bar{b}_{i}^{\prime}=\bar{b}_{i}$ and

$$
\sum_{i=1}^{m} a_{i} b_{i}^{\prime}=0 \quad(\text { since } B \text { is antisymmetric) } .
$$

Let $b_{i}^{\prime \prime}=b_{\imath}-b_{i}^{\prime}$ with $b_{i}^{\prime}=0$ for $i>m$. We have: $\sum_{i=1}^{k} a_{i} b_{i}^{\prime \prime}=0$ by (1.4.1) and (1.4.5), and $\max _{i} \operatorname{deg} a_{i} b_{i}^{\prime \prime}<d$. Since $b_{\imath}^{\prime} \in\left(a_{1}, \ldots, a_{i-1}, a_{i+1}, \ldots\right)$ by (1.4.4) (recall that the diagonal entries of $B$ are zero) we obtain a contradiction with (1.4.1).

The proof of (b) is similar. Let $x \in I$, if we can find an expression $x=\sum_{i=1}^{n} a_{1} b_{1}$ such that, setting $d=\max _{i} \operatorname{deg} a_{i} b_{i}$, we have $d=\operatorname{deg} x$, we are clearly done. Suppose this is not the case and choose an expression for which $d>\operatorname{deg} x$ is minimal. As in (1.4.2) assume $\operatorname{deg} a_{\imath} b_{\imath}=d$ for $i=1, \ldots, m$ while $\operatorname{deg} a_{i} b_{i}<d$ for $i>m$. Thus we have $\sum_{i=1}^{m} \bar{a}_{i} \bar{b}_{i}=0$ and as before we can find $b_{1}^{\prime}, \ldots, b_{m}^{\prime}$ such that $\sum_{i=1}^{m} a_{i} b_{i}^{\prime}=0$ and $\operatorname{deg} a_{i}\left(b_{i}-b_{i}^{\prime}\right)<d$ for $i=1, \ldots, m$, reaching a contradiction.

1.5. Let $A$ be an order closed under trace in a central simple algebra $D$, let $Z$ be the center of $A$ and $Q(Z)$ that of $D$ as in Sect. 1.1.

Definition 1.5. A trace filtration for $A$ is a filtration $A_{i}$ such that:

(a) $\operatorname{tr}\left(A_{i}\right) \subset A_{i}$.

(b) $\bar{A}$ is finitely generated.

Proposition 1.5. Let $A$ be an order in $D$ closed under trace and with a trace filtration, then $\mathscr{R}(A)$ is a finitely generated order in $D(t)$ closed under trace.

Proof. By Lemma $1.3 \mathrm{c}$ it follows immediately that $\mathscr{R}(A)$ is an order in $D(t)$, but by Lemma $1.3 \mathrm{~b}$ both $A$ and $\mathscr{R}(A)$ are finitely generated. The assumptions on the filtration imply that $\mathscr{R}(A)$ is closed under trace.

The following simple lemma is of crucial importance for this paper. 
Lemma 1.5. Let $A$ be a filtered algebra such that $\bar{A}$ is a finitely generated order of the same degree as $A$, and let $Z_{0}$ be a central subalgebra of $A$ such that $\bar{Z}_{0}$ is finitely generated and $\bar{A}$ is a finitely generated module over $\bar{Z}_{0}$. Let $I$ be an ideal of $Z_{0}$ and $\bar{I}$ the associated graded ideal of $\bar{Z}_{0}$. Let $\odot$ (resp. $\left.O_{1}\right)$ be the set of zeros of $I$ (resp. $\left.\bar{I}\right)$ in $\operatorname{Spec} Z_{0}$ (resp. Spec $\bar{Z}_{0}$ ). Suppose that $\mathscr{Q}_{1} \cap \Omega_{\bar{A}}^{0} \neq \emptyset$. Then $\mathcal{O} \cap \Omega_{A}^{0} \neq \emptyset$.

Proof. Consider the Rees algebra $\mathscr{R}(A)$ of $A$. Its subalgebra $\mathscr{R}\left(Z_{0}\right)$ is central and by Proposition $1.3, \mathscr{R}(A)$ is a finitely generated $\mathscr{R}\left(Z_{0}\right)$-module. We have seen that

$$
\text { degree } \mathscr{B}(A)=\text { degree } A
$$

and by the hypothesis:

$$
\operatorname{degree}(\bar{A})=\operatorname{degree} A
$$

hence

Clearly

$$
\Omega_{\mathscr{R}(A)}^{0} \cap \operatorname{Spec} \bar{Z}_{0}=\Omega_{\bar{A}}^{0} .
$$

$$
\Omega_{\mathscr{B}(A)}^{0} \supset \Omega_{A\left[t, t^{-1}\right]}^{0}=\Omega_{A}^{0} \times \mathbb{F}^{\times},
$$

and by Proposition 1.4 we have:

$$
\left(\overline{\left(\sigma \times \mathbb{F}^{\times}\right.}\right) \cap \operatorname{Spec} \bar{Z}_{0}=Q_{1} \text {. }
$$

Hence $\Omega_{\mathscr{R}(A)}^{0} \cap\left(\overline{\mathscr{O}^{\circ} \times \mathbb{F}^{\times}}\right) \supset \Omega_{\bar{A}}^{0} \cap\left(\overline{\left(\times \mathbb{F}^{\times}\right.}\right) \neq \emptyset$ (where $\overline{\mathscr{O} \times \mathbb{F}^{\times}}$stands for Zariski closure of $\bigcirc \times \mathbb{F}^{\times}$) since $Q_{1} \cap \Omega_{\bar{A}}^{0} \neq \emptyset$ by the hypothesis. It follows that $\Omega_{\mathscr{R}(A)}^{0}$ intersects with $\odot \times \mathbb{F}^{\times}$in a non-empty open subset. But, obviously, this intersection is $\left(\bigcirc \cap \Omega_{A}^{0}\right) \times \mathbb{F}^{\times}$. It follows that $\bigcirc \cap \Omega_{A}^{0} \neq \emptyset$.

\section{Representation Theory of Twisted Polynomial Algebras}

2.1. Let $A$ be an algebra and let $\sigma$ be an automorphism of $A$. The twisted polynomial algebra $A_{\sigma}[x]$ in the indeterminate $x$ is the $\mathbb{F}$-module $A \otimes_{\mathbb{F}} \mathbb{F}[x]$ with multiplication

$$
\left(a \otimes x^{m}\right)\left(b \otimes x^{n}\right)=a \sigma^{m}(b) \otimes x^{m+n} .
$$

We may similarly consider the twisted Laurent polynomial algebra $A_{\sigma}\left[x, x^{-1}\right]$. It is clear that if $A$ has no zero divisors, then the algebras $A_{\sigma}[x]$ and $A_{\sigma}\left[x, x^{-1}\right]$ also have no zero divisors.

Lemma 2.1. If $M$ is an irreducible module over $A_{\sigma}[x]$, then there are two possibilities:

(i) $x=0$, hence $M$ is actually an A-module,

(ii) $x$ is invertible, hence $M$ is actually an $A_{\sigma}\left[x, x^{-1}\right]$-module.

Proof. It is clear that $\operatorname{Im}(x)$ and $\operatorname{Ker}(x)$ are submodules of $M$.

2.2. Let $\mathbb{F}$ be a field and $q \in \mathbb{F}^{\times}$a given element. Given an $n \times n$ skewsymmetric matrix $H=\left(h_{i j}\right)$ over $\mathbb{Z}$, we construct the twisted polynomial algebra $\mathbb{F}_{H}\left[x_{1}, \ldots, x_{n}\right]$. This is the algebra on generators $x_{1}, \ldots, x_{n}$ and the following defining relations:

$$
x_{\imath} x_{j}=q^{h_{i j}} x_{j} x_{\imath} \quad(i, j=1, \ldots, n) .
$$

It can be viewed as an iterated twisted polynomial algebra with respect to any ordering of the indeterminates $x_{\imath}$. Similarly, we can define the twisted Laurent polynomial algebra $\mathbb{F}_{H}\left[x_{1}, x_{1}^{-1}, \ldots, x_{n}, x_{n}^{-1}\right]$. Both algebras have no zero divisors. 
As a consequence of Lemma 2.1, we have

Lemma 2.2. In any irreducible $\mathbb{F}_{H}\left[x_{1}, \ldots, x_{n}\right]$-module each element $x_{i}$ is either 0 or invertible.

Given $a=\left(a_{1}, \ldots, a_{n}\right) \in \mathbb{Z}^{n}$, we shall write $x^{a}=x_{1}^{a_{1}} \ldots x_{n}^{a_{n}}$. The torus $\mathbb{F}^{\times n}$ acts by automorphisms of the algebra $\mathbb{F}_{H}\left[x_{1}, \ldots, x_{n}\right]$ and $\mathbb{F}_{H}\left[x_{1}, x_{1}^{-1}, \ldots, x_{n}, x_{n}^{-1}\right]$ in the usual way, the monomial $x^{a}$ being a weight vector of weight $a$. Consider the group $G$ of inner automorphisms of the Laurent polynomials generated by conjugation by the variables $x_{i}$. Clearly $G$ induces a group of automorphisms of the twisted polynomial algebra which are in this torus of automorphisms. In fact one can formalize this as follows: Let $\Gamma:=\left\{\alpha x^{a} \mid \alpha \in \mathbb{F}^{\times}\right\}$be the set of non-zero monomials. Then $\Gamma$ is a group, $\mathbb{F}^{\times}$is a central subgroup and $\Gamma / \mathbb{F}^{\times}$is free abelian, the homomorphism $\Gamma \rightarrow\left(\mathbb{F}^{\times}\right)^{n}$ given by considering the associated inner automorphisms has as kernel the monomials in the center.

Let $\varepsilon$ be a primitive $\ell^{\text {th }}$ root of 1 in $\mathbb{F}$ and take now $q=\varepsilon$. We consider the matrix $H$ as a matrix of a homomorphism $H: \mathbb{Z}^{n} \rightarrow(\mathbb{Z} / \ell \mathbb{Z})^{n}$, and we denote by $K$ the kernel of $H$ and by $h$ the cardinality of the image of $H$.

Proposition 2.2. (a) The elements $x^{a}$ with $a \in K \cap \mathbb{Z}_{+}^{n}$ (resp. $a \in K$ ) form a basis of the center of $\mathbb{F}_{H}\left[x_{1}, \ldots, x_{n}\right]$ (resp. $\mathbb{F}_{H}\left[x_{1}, x_{1}^{-1}, \ldots, x_{n}, x_{n}^{-1}\right]$ ).

(b) Let $a^{(1)}, \ldots, a^{(h)}$ be a set of representatives of $\mathbb{Z}^{n} \bmod K$. Then the monomials $x^{a^{(1)}}, \ldots, x^{a^{(h)}}$ form a basis of the algebra $\mathbb{F}_{H}\left[x_{1}, x_{1}^{-1}, \ldots, x_{n}, x_{n}^{-1}\right]$ over its center. (c) degree $\mathbb{F}_{H}\left[x_{1}, \ldots, x_{n}\right]=$ degree $\mathbb{F}_{H}\left[x_{1}, x_{1}^{-1}, \ldots, x_{n}, x_{n}^{-1}\right]=\sqrt{h}$.

Proof. Define a skewsymmetric bilinear form on $\mathbb{Z}^{n}$ by letting for $a=\left(a_{1}, \ldots, a_{n}\right)$, $b=\left(b_{1}, \ldots, b_{n}\right) \in \mathbb{Z}^{n}:\langle a \mid b\rangle=\sum_{i, j=1}^{n} h_{i j} a_{i} b_{j}$. Then we have

$$
x^{a} x^{b}=\varepsilon^{\langle a \mid b\rangle} x^{b} x^{a} .
$$

Since the center is invariant with respect to the action of $\mathbb{F}^{\times n}$, it must have a basis of elements of the form $x^{a}$. This together with (2.2.1) implies (a).

(b) follows from (a) and the fact that

$$
x^{a} x^{b}=\varepsilon^{c(a, b)} x^{a+b}, \text { where } c(a, b)=\sum_{\imath>j} h_{i j} a_{i} b_{\jmath} .
$$

(c) follows from (b).

It is quite easy to see that the twisted polynomial algebras we are considering are closed under trace $[\mathrm{DC}-\mathrm{K}]$ and in fact from (2.2.1) one can easily deduce a formula for the trace

$$
\operatorname{tr}\left(x^{a}\right)=0 \text { if } x^{a} \text { is not in the center } .
$$

2.3. Recall that an algebra $A$ over a commutative $\operatorname{ring} Z$ is called an Azumaya algebra of degree $d$ if there exists a faithfully flat ring extension $Z^{\prime}$ of $Z$ such that $A \otimes Z^{\prime}$ is isomorphic to the full algebra of $d \times d$ matrices over $Z^{\prime}$.

Suppose now that $\mathbb{F}$ is an algebraically closed field and that $A$ is a prime algebra over $\mathbb{F}$ (i.e. $a A b=0, a, b \in A$ implies $a=0$ or $b=0$ ), which is finitely generated module over a finitely generated subalgebra $Z_{0}$ of the center $Z$. Then $A$ is an Azumaya 
algebra over $Z$ if and only if all irreducible representations of $A$ have the same dimension $(=d)$ [A-P1].

Proposition 2.3. Let $\mathbb{F}$ be an algebraically closed field. Then any Laurent quasipolynomial algebra $\mathbb{F}_{H}\left[x_{1}, x_{1}^{-1}, \ldots, x_{n}, x_{n}^{-1}\right]$ is an Azumaya algebra over its center. In particular, all irreducible representations of the algebra $\mathbb{F}_{H}\left[x_{1}, \ldots, x_{n}\right]$ for which all $x_{i} \neq 0$ have dimension $\sqrt{h}$.

Proof. Let $Z_{0}=\left\{x^{a} \mid a \in(\ell \mathbb{Z})^{n}\right\}$. This is a finitely generated central subalgebra over which the algebra $A:=\mathbb{F}_{H}\left[x_{1}, x_{1}^{-1}, \ldots, x_{n}, x_{n}^{-1}\right]$ is finitely generated. Recall that we have the surjective map $\chi_{0}: \operatorname{Spec} A \rightarrow \operatorname{Spec} Z_{0}$ and that the set $\Omega_{A}^{0}=\left\{a \in \operatorname{Spec} Z_{0} \mid \chi_{0}^{-1}(a)\right.$ consists of representations of maximal dimension $\}$ is a dense open subset of $\operatorname{Spec} Z_{0}$ (see Sect. 1.2). But the group $\mathbb{F}^{\times n}$ of automorphisms of $A$ acts transitively on $\operatorname{Spec} Z_{0}$, hence $\Omega_{A}^{0}=\operatorname{Spec} Z_{0}$, proving the proposition.

2.4. Let $A:=\mathbb{F}_{H}\left[x_{1}, \ldots, x_{n}\right]$ be as in Sect. 2.2 (of degree $\sqrt{h}$ ). Recall that the torus $T:=\mathbb{F}^{\times n}$ acts by automorphisms of $A$ and hence of its center, so that the representation picture looks like a non-commutative version of affine torus embeddings. First of all remark that, by Lemma 2.2, the vanishing of the central element $x_{i}^{l}$ in an irreducible representation implies the vanishing of $x_{i}$. Thus it is natural to stratify the Spec $A$ according to the set $S$ of indices $i$ for which $x_{i}^{l} \neq 0$ and remark that this stratification is just the stratification by orbits under $T$. Let $A_{S}$ denote the twisted Laurent polynomial algebra in the variables $x_{i}, i \in S$. From Sect. 2.3 we have that $A_{S}$ is an Azumaya algebra whose degree $d_{S}$ is computed as in Proposition 2.2 by restricting the homomorphism $H$ to the subgroup of $\mathbb{Z}^{n}$ formed by the vectors with zero coordinates in the indices not in $S$, i.e. by analyzing the skew submatrix $H_{S}$ of $H$ which defines $A_{S}$. The spectrum of its center is isomorphic to a quotient $T_{S}$ of the torus $T$.

On the other hand we can pass from $A$ to $A_{S}$ as follows. First we can invert in $A$ the elements $x_{\imath}, i \in S$, to get an algebra which we may call $A_{S}^{\prime}$. In $A_{S}^{\prime}$ we have the ideal $I_{S}$ generated by the variables $x_{\imath}, i \notin S$, and we clearly have that $A_{S}=A_{S}^{\prime} / I_{S}$. The center of $A_{S}^{\prime}$ is the center of $A$ localized at the elements $x_{i}^{l}$ and its points parametrize equivalence classes of semisimple representations of degree $\sqrt{h}$, where the central character is non-zero in the $x_{i}^{l}, i \in S$. The algebra $A_{S}$ inherits from $A_{S}^{\prime}$ a trace map tr with values in the quotient $Z_{S}^{\prime}$ of the center of $A_{S}^{\prime}$ by the ideal generated by the elements $x_{\imath}, i \in S$. It is not hard to see that the picture is the following. In $A_{S}$ we have the center $Z_{S}$ and its subring $Z_{S}^{\prime}$ over which $Z_{S}$ is finite. The spectrum of $Z_{S}^{\prime}$ is also isomorphic to a quotient $T_{S}^{\prime}$ of the torus $T$ and $T_{S}^{\prime}$ is a quotient $T_{S} / \Gamma$ by a finite subgroup $\Gamma$. In particular each fiber of the map $\pi: \operatorname{Spec} Z_{S} \rightarrow \operatorname{Spec} Z_{S}^{\prime}$ is reduced and consists of a coset of the finite group $\Gamma$. We have several trace maps: the reduced trace $\operatorname{tr}_{A_{S} / Z_{S}}$ to the center, the trace of the finite map $\operatorname{tr}_{Z_{S} / Z_{S}^{\prime}}$ and the composition $\operatorname{tr}_{A_{S} / Z_{S}^{\prime}}$. From (2.2.3) and the torus description it follows that there exists a positive integer $d$ such that $\operatorname{tr}=d \operatorname{tr}_{A_{S} / Z_{S}^{\prime}}$. From this an Sect. 1.1 it follows that each point of the spectrum of $Z_{S}^{\prime}$ corresponds to a semisimple representation which is obtained counting with multiplicity $d$ each irreducible representations of $A_{S}$ appearing in the fiber of the map $\pi: \operatorname{Spec} Z_{S} \rightarrow \operatorname{Spec} Z_{S}^{\prime}$. Of course we have: $d|\Gamma| d_{S}=\sqrt{h}$.

This program will be developed in Sect. 3 for a particular skew-symmetric matrix arising from root systems which, as we shall see in Sects. 4,5 gives rise to the twisted polynomial ring to which the quantum group degenerates. 


\section{Some Properties of Finite Root Systems}

3.1. Let $\left(a_{i j}\right)$ be a $n \times n$-matrix with integer entries such that $(i, j=1, \ldots, n)$ :

$$
a_{i i}=2, a_{i j} \leq 0 \text { if } i \neq j,
$$

and there exists a vector $\left(d_{1}, \ldots, d_{n}\right)$ with relatively prime positive integral entries $d_{i}$ such that

$$
\left(d_{i} a_{i j}\right) \text { is a symmetric positive definite matrix. }
$$

Of course $\left(a_{i j}\right)$ is a Cartan matrix, to which there is associated finite reduced root system $R$, its weight and root lattices $P$ and $Q$, the Weyl group $W$, a set of positive roots $R^{+}$, the set of simple roots $\Pi$, the fundamental weights $\omega_{1}, \ldots, \omega_{n}$, etc. Let us recall for convenience the basic definitions.

Let $P$ be a lattice over $\mathbb{Z}$ with basis $\omega_{1}, \ldots, \omega_{n}$ and let $Q^{\vee}=\operatorname{Hom}_{\mathbb{Z}}(P, \mathbb{Z})$ be the dual lattice with dual basis $\alpha_{1}^{\vee}, \ldots, \alpha_{n}^{\vee}$, i.e. $\left\langle\omega_{i}, \alpha_{j}^{\vee}\right\rangle=\delta_{i j}$. Let $P_{+}=\sum_{i=1}^{n} \mathbb{Z}_{+} \omega_{i}$. Let

$$
\rho=\sum_{i=1}^{n} \omega_{i}, \alpha_{\jmath}=\sum_{i=1}^{n} a_{i j} \omega_{\imath} \quad(j=1, \ldots, n),
$$

and let $Q=\sum_{j=1}^{n} \mathbb{Z} \alpha_{j} \subset P$, and $Q_{+}=\sum_{j=1}^{n} \mathbb{Z}_{+} \alpha_{j}$. Define the usual partial ordering on $P$ by $\lambda \geq \mu$ if $\lambda-\mu \in Q_{+}$. For $\beta=\sum_{i} k_{i} \alpha_{i} \in Q$ let $h t \beta=\sum_{i} k_{i}$.

Define automorphisms $s_{i}$ of $P$ by $s_{i}\left(\omega_{j}\right)=\omega_{j}-\delta_{i j} \alpha_{i}(i, j=1, \ldots, n)$. Then $s_{\imath}\left(\alpha_{j}\right)=\alpha_{j}-a_{i j} \alpha_{\imath}$. Let $W$ be the subgroup of $G L(P)$ generated by $s_{1}, \ldots, s_{n}$. Let

$$
\begin{aligned}
& \Pi=\left\{\alpha_{1}, \ldots, \alpha_{n}\right\}, \Pi^{\vee}=\left\{\alpha_{1}^{\vee}, \ldots, \alpha_{n}^{\vee}\right\}, \\
& R=W \Pi, R^{+}=R \cap Q_{+}, R^{\vee}=W \Pi^{\vee} .
\end{aligned}
$$

The map $\alpha_{i} \longmapsto \alpha_{i}^{\vee}$ extends uniquely to a bijective $W$-equivariant map $\alpha \longmapsto \alpha^{\vee}$ between $R$ and $R^{\vee}$. The reflection $s_{\alpha}$ defined by $s_{\alpha}(\lambda)=\lambda-\left\langle\lambda, \alpha^{\vee}\right\rangle \alpha$ lies in $W$ for each $\alpha \in R$, so that $s_{\alpha_{i}}=s_{i}$.

Define a bilinear pairing $P \times Q \rightarrow \mathbb{Z}$ by $\left(\omega_{i} \mid \alpha_{j}\right)=\delta_{i j} d_{j}$. Then $\left(\alpha_{\imath} \mid \alpha_{j}\right)=d_{i} \alpha_{i j}$, giving a symmetric $\mathbb{Z}$-valued $W$-invariant bilinear form on $Q$ such that $(\alpha \mid \alpha) \in 2 \mathbb{Z}$. We may identify $Q^{\vee}$ with a sublattice of the $\mathbb{Q}$-span of $P$ (containing $Q$ ) using this form. Then:

$$
\alpha_{\imath}^{\vee}=d_{\imath}^{-1} \alpha_{i}, \quad \alpha^{\vee}=2 \alpha /(\alpha \mid \alpha) \text {. }
$$

3.2. Let now $\omega_{0}$ be the longest element of $W$ so that $\omega_{0}\left(R^{+}\right)=-R^{+}, \omega_{0}(\Pi)=-\Pi$ and $\omega_{0}\left(P_{+}\right)=-P_{+}$. For $\alpha \in R^{+}$(resp. $\lambda \in P_{+}$) we let ${ }^{t} \alpha=-\omega_{0}(\alpha) \in R^{+}$ (resp. ${ }^{t} \lambda=-\omega_{0}(\lambda)$ ). For a fundamental weight $\omega$, the weight ${ }^{t} \omega$ is also fundamental. Fix a reduced expression

$$
\omega_{0}=s_{i_{1}} s_{i_{2}} \ldots s_{i_{N}}, \text { where } N=\left|R^{+}\right| .
$$

and consider the corresponding convex ordering of $R^{+}$:

$$
\beta_{1}=\alpha_{i_{1}}, \beta_{2}=s_{\imath_{1}}\left(\alpha_{i_{2}}\right) \ldots, \beta_{N}=s_{i_{1}} \ldots s_{\imath_{N-1}}\left(\alpha_{i_{N}}\right) .
$$

(The name "convex" refers to the property that if $i<j$ and $\beta_{i}+\beta_{j} \in R^{+}$, then $\beta_{i}+\beta_{j}=\beta_{k}$ for some $k$ between $i$ and $j$.) 
Given a simple root $\alpha \in \Pi$, let

$$
I_{\alpha}=\left\{t \in \mathbb{Z} \mid 1 \leq t \leq N, s_{\imath_{t}}=s_{\alpha}\right\},
$$

so that the set $\{1,2, \ldots, N\}$ is a disjoint union of the sets $I_{\alpha}$.

Lemma 3.2. Fix a simple root $\alpha$ and let $\omega$ be the corresponding fundamental weight. Let $k_{1}<\ldots<k_{r}$ be all elements of the set $I_{\alpha}$. For $t \in \mathbb{Z}, 0 \leq t \leq r$, let

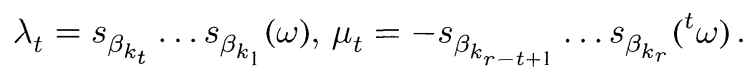

Then:

(a) $\lambda_{t}=s_{i_{1}} s_{\imath_{2}} \ldots s_{i_{k_{t}}}(\omega)$; in particular $\lambda_{r}=-{ }^{t} \omega$.

(b) If $k_{t}<j<k_{t+1}$, then $\left\langle\lambda_{t}, \beta_{j}^{\vee}\right\rangle=0$.

(c) $\left\langle\lambda_{t}, \beta_{k_{t+1}}^{\vee}\right\rangle=1$.

(d) $\lambda_{t}=\omega-\sum_{i=1}^{t} \beta_{k_{i}}$.

Similarly:

(a') $\mu_{t}=\lambda_{r-t}$; in particular, $\mu_{r}=\omega$.

(b') If $k_{r-t}<j<k_{r-t+1}$, then $\left\langle\mu_{t}, \beta_{j}^{\vee}\right\rangle=0$.

(c') $\left\langle\mu_{t}, \beta_{k_{r-t}}^{\vee}\right\rangle=-1$.

$\left(\mathrm{d}^{\prime}\right)-s_{\beta_{k_{1}}} \ldots s_{\beta_{k_{r}}}\left({ }^{t} \omega\right)=-{ }^{t} \omega+\sum_{i=1}^{r} \beta_{k_{\imath}}=\omega$.

Proof. If $k_{t}<j<k_{t+1}$, then

$$
\begin{aligned}
\lambda_{t}= & s_{\beta_{k_{t}} \ldots s_{\beta_{k_{1}}}}(\omega)=\left(s_{i_{1}} \ldots s_{i_{k_{t}-1}} s_{\imath_{k_{t}}} s_{i_{k_{t}-1}} \ldots s_{\imath_{1}}\right) \\
& \times\left(s_{\imath_{1}} \ldots s_{i_{k_{t-1}-1}} s_{i_{k_{t-1}}} s_{i_{k_{t-1}-1}} \ldots s_{\imath_{1}}\right) \ldots\left(s_{i_{1}} \ldots s_{i_{k_{1}-1}} s_{\imath_{k_{1}}} s_{i_{k_{1}-1}} \ldots s_{\imath_{1}}\right) \\
= & s_{\imath_{1}} \ldots s_{i_{k_{1}}} \tilde{w}(\omega)
\end{aligned}
$$

where $\tilde{w}$ doesn't contain $s_{\alpha}$. This proves (a).

Hence

$$
\begin{aligned}
\left\langle\lambda_{t}, \beta_{j}^{\vee}\right\rangle & =\left\langle s_{i_{1}} \ldots s_{\imath_{k_{t}}}(\omega), s_{i_{1}} \ldots s_{\imath_{j-1}}\left(\alpha_{j}^{\vee}\right)\right\rangle=\left\langle s_{\imath_{\jmath}-1} \ldots s_{i_{k_{t}+1}}(\omega), \alpha_{\jmath}^{\vee}\right\rangle \\
& =\left\langle\omega, \alpha_{\jmath}^{\vee}\right\rangle=0,
\end{aligned}
$$

proving (b).

By (a) we have:

$$
\begin{aligned}
\left\langle\lambda_{t}, \beta_{k_{t+1}}^{\vee}\right\rangle & =\left\langle s_{i_{1}} \ldots s_{\imath_{k_{t}}}(\omega), s_{i_{1}} \ldots s_{\imath_{k_{t+1}-1}} \alpha^{\vee}\right\rangle=\left\langle s_{k_{t+1}-1} \ldots s_{k_{t}+1}(\omega), \alpha^{\vee}\right\rangle \\
& =\left\langle\omega, \alpha^{\vee}\right\rangle=1,
\end{aligned}
$$

proving (c). (d) follows from (c).

Furthermore, we have:

$$
\mu_{t}=s_{\beta_{k_{r-t+1}}} \ldots s_{\beta_{k_{r}}} s_{i_{1}} \ldots s_{i_{N}}(\omega) .
$$

As in the proof of (a), replacing in this equality each $s_{\beta_{k}}$ by the corresponding conjugate of $s_{\imath_{k}}$, we obtain $\mu_{t}=s_{\imath_{1}} \ldots s_{i_{k_{r-t}}} \tilde{w}(w)$, where $\tilde{w}$ does not contain $s_{\alpha}$. Hence $\mu_{t}=s_{\imath_{1}} \ldots s_{i_{k_{r-t}}}(\omega)=\lambda_{r-t}$ by (a). 
Now (b') follows from (b) and (d') from (d). Finally, $\left\langle\mu_{t}, \beta_{k_{r-t}}^{\vee}\right\rangle=\left\langle\lambda_{r-t}, \beta_{k_{r-t}}^{\vee}\right\rangle$ $=\left\langle s_{\beta_{k_{r-t}}} \lambda_{r-t-1}, \beta_{k_{r-t}}^{\vee}\right\rangle=-\left\langle\lambda_{r-t-1}, \beta_{k_{r-t}}^{\vee}\right\rangle=-1$ by (b), proving $\left(\mathrm{c}^{\prime}\right)$.

Corollary 3.2. (a) $\left\langle\beta_{\ell}^{\vee}, \omega\right\rangle=\sum_{k_{\imath}<\ell}\left\langle\beta_{\ell}^{\vee}, \beta_{k_{i}}\right\rangle$ if $\ell \notin I_{\alpha}$.

(b) $\left\langle\beta_{k_{t}}^{\vee}, \omega\right\rangle=1+\sum_{i<t}\left\langle\beta_{k_{t}}^{\vee}, \beta_{k_{i}}\right\rangle$ if $k_{t} \in I_{\alpha}$.

Proof. By Lemma 3.2a,d we have:

$\left\langle\beta_{\ell}^{\vee}, \omega-\sum_{k_{\imath}<\ell} \beta_{k_{i}}\right\rangle=\left\langle s_{i_{1}} \ldots s_{i_{\ell-1}}\left(\alpha_{i_{\ell}}^{\vee}\right), s_{i_{1}} \ldots s_{i_{\ell-1}}(\omega)\right\rangle=\left\langle\alpha_{\ell_{\ell}}^{\vee}, \omega\right\rangle=0$, proving (a).

Similarly, $\left\langle\beta_{k_{t}}^{\vee}, \omega-\sum_{i<t} \beta_{k_{i}}\right\rangle=\left\langle\alpha^{\vee}, \omega\right\rangle=1$.

3.3. Consider the free $\mathbb{Z}\left[\frac{1}{2}\right]$-modules $V_{+}$with basis $\varphi_{\beta_{1}}, \ldots, \varphi_{\beta_{n}}, V_{-}$with basis $\varphi_{-\beta_{1}}, \ldots, \varphi_{-\beta_{N}}$ and $V_{0}$ with basis $\omega_{1}, \ldots, \omega_{n}$. On the $\mathbb{Z}\left[\frac{1}{2}\right]$-module $V=V_{+} \oplus$ $V_{-} \oplus V_{0}$ define a skew symmetric bilinear form $\langle. \mid$.$\rangle by the following formulas:$

$$
\left\{\begin{array}{l}
\left\langle V_{-} \mid V_{+}\right\rangle=0,\left\langle V_{0} \mid V_{0}\right\rangle=0 \\
\left\langle\varphi_{\beta_{i}} \mid \varphi_{\beta_{j}}\right\rangle=-\left\langle\varphi_{-\beta_{i}} \mid \varphi_{-\beta_{\jmath}}\right\rangle=\left(\beta_{i} \mid \beta_{j}\right) \text { if } i<j \\
\left\langle\omega_{i} \mid \varphi_{\beta_{\jmath}}\right\rangle=-\left\langle\omega_{i} \mid \varphi_{-\beta_{\jmath}}\right\rangle=\left(\omega_{i} \mid \beta_{j}\right)
\end{array}\right.
$$

Introduce the matrices:

$$
A=\left(\left\langle\varphi_{\beta_{\imath}} \mid \varphi_{\beta_{\jmath}}\right\rangle\right)_{1 \leq i, j \leq N}, B=\left(\left(\omega_{i} \mid \beta_{\jmath}\right)\right)_{1 \leq \imath \leq n, 1 \leq j \leq N}
$$

Then the matrix $S$ of the skew symmetric form $\langle$.$| . \rangle$ in the above basis has the form:

$$
S=\left(\begin{array}{rrr}
A & 0 & -{ }^{t} B \\
0 & -A & { }^{t} B \\
B & -B & 0
\end{array}\right)
$$

Proposition 3.3. All non-zero elementary divisors of the matrix $S$ over the ring $\mathbb{Z}\left[\frac{1}{2}\right]$ are $\frac{1}{2}\left(\beta_{i} \mid \beta_{\imath}\right), i=1, \ldots, N$, each repeated twice.

Proof. Consider the matrix

$$
C=\left(\begin{array}{rrr}
I_{N} & I_{N} & 0 \\
-\frac{1}{2} I_{N} & \frac{1}{2} I_{N} & 0 \\
0 & 0 & I_{N}
\end{array}\right) \in G L\left(2 N+n, \mathbb{Z}\left[\frac{1}{2}\right]\right)
$$

Let $S^{\prime}=C S^{t} C$. We have:

$$
S^{\prime}=\left(\begin{array}{rrr}
0 & -A & 0 \\
-A & 0 & { }^{t} B \\
0 & -B & 0
\end{array}\right)
$$

Since $S^{\prime}=S_{1}-{ }^{t} S_{1}$, where $S_{1}=\left(\begin{array}{rrr}0 & 0 & 0 \\ -A & 0 & { }^{t} B \\ 0 & 0 & 0\end{array}\right)$, we see that $S_{1}(V) \subset$ $V_{-}, t_{1}^{S}(V) \subset V_{+} \oplus V_{0}$. Hence it suffices to show that all non-zero elementary divisors 
of the matrix $S_{1}$ over $\mathbb{Z}\left[\frac{1}{2}\right]$ are $\frac{1}{2}\left(\beta_{i} \mid \beta_{\imath}\right), i=1, \ldots, N$. Note that

$$
\begin{aligned}
S_{1}\left(\varphi_{\beta_{1}}\right) & =\sum_{\jmath} \operatorname{sign}(j-i)\left(\beta_{\imath} \mid \beta_{\jmath}\right)_{\varphi_{-\beta_{j}}} \\
S_{1}\left(\beta_{i}\right) & =-\sum_{\jmath}\left(\beta_{\imath} \mid \beta_{j}\right) \varphi_{-\beta_{\jmath}} .
\end{aligned}
$$

Hence we have:

$$
S_{1}\left(\varphi_{\beta_{\imath}}+\beta_{i}\right)=-\left(\beta_{i} \mid \beta_{i}\right)_{\varphi_{-\beta_{i}}}-2 \sum_{j<i}\left(\beta_{i} \mid \beta_{j}\right) \varphi_{-\beta_{j}}
$$

Since $\left(\beta_{\imath} \mid \beta_{j}\right)$ is divisible by $\frac{1}{2}\left(\beta_{i} \mid \beta_{i}\right)$, this completes the proof.

Lemma 3.3. (a) The vectors $v_{\omega}=\sum_{t \in I_{\alpha}}\left(\varphi_{\beta_{t}}+\varphi_{-\beta_{t}}\right)+\omega-{ }^{t} \omega$ form a basis of the kernel of the form $\langle. \mid$.$\rangle as \omega$ runs over all fundamental weights.

(b) Suppose that $\ell$ is a positive odd integer relatively prime to all the $d_{i}$. Then the kernel of the map $S: \mathbb{Z}^{2 N+n} \rightarrow(\mathbb{Z} / \ell \mathbb{Z})^{2 N+n}$ is spanned by vectors $v_{\omega_{\imath}}(i=1, \ldots, n)$ and $\ell \mathbb{Z}^{2 N+n}$. Its image is isomorphic to $(\mathbb{Z} / \ell \mathbb{Z})^{2 N}$.

(c) Select in each set $I_{\alpha_{i}}$ a root $\beta_{t_{i}}$ and consider the submodule $V^{\prime}$ of $V$ of the vectors which have coordinate 0 for the corresponding vectors $\varphi_{\beta_{t_{i}}}$ or $\varphi_{-\beta_{t_{i}}}$, and call $S^{\prime}$ the restriction of $S$ to $V^{\prime}$. Then the kernel of $S^{\prime}$ coincides with $\ell V^{\prime}$ and $S^{\prime}\left(V^{\prime}\right)=S(V)$.

Proof. Due to Proposition 3.3, to prove (a), it suffices to show that the elements $v_{\omega}$ lie in the kernel. We have (using Lemma $3.2 \mathrm{~d}^{\prime}$ ):

$$
\begin{aligned}
\left\langle\varphi_{\beta_{j}} \mid v_{\omega}\right\rangle & =-\sum_{k_{t}<j}\left(\beta_{j} \mid \beta_{k_{t}}\right)+\sum_{k_{t}>\jmath}\left(\beta_{\jmath} \mid \beta_{k_{t}}\right)+2\left(\beta_{j} \mid \omega\right)-\sum_{k_{t}}\left(\beta_{\jmath} \mid \beta_{k_{t}}\right) \\
& =2\left(\beta_{j} \mid \omega-\sum_{k_{t} \leq \jmath} \beta_{k_{t}}\right)- \begin{cases}\left(\beta_{j} \mid \beta_{j}\right) & \text { if } j \in I_{\alpha} \\
0 & \text { otherwise }\end{cases} \\
& =0 \text { by Corollary } 3.2 \text { and (3.1.3). }
\end{aligned}
$$

Similarly, $\left\langle\varphi_{-\beta_{j}} \mid v_{\omega}\right\rangle=0$ and trivially $\left\langle v \mid v_{\omega}\right\rangle=0$ for $v \in V_{0}$. This proves part (a) of the proposition, (b) and (c) follow easily.

Remark 3.3. Suppose that $\left(a_{\imath \jmath}\right)$ is an indecomposable Cartan matrix. Then all the $d_{\imath}$ are equal 1 or 2 except for the case $\left(a_{i j}\right)$ is of type $G_{2}$. In the latter case $d_{1}=3$, $d_{2}=1$ and if $\ell$ is divisible by 3 , the kernel of the map $S$ is spanned by vectors $v_{\omega_{i}}(i=1,2), \frac{1}{3} \ell \varphi_{\beta}(\beta$ long root in $R)$, and $\ell \mathbb{Z}^{14}$.

\section{Some Properties of the Central Subalgebra $Z_{0}$ of $U_{\varepsilon}$.}

4.1. Let $\left(a_{i j}\right)$ be an $n \times n$ Cartan matrix (as in Sect. 3), $P$ its weight lattice and $q$ an indeterminate. One defines (cf. [DC-K-P] to which we refer for all the necessary notations) the simply connected quantum group $U$ associated to the matrix $\left(a_{\imath \jmath}\right)$ as a Hopf algebra over $\mathscr{b}:=\mathbb{C}\left[q, q^{-1},\left(q^{d_{\imath}}-q^{-d_{\imath}}\right)^{-1}\right]$ on generators $E_{i}, F_{i}(i=$ $1, \ldots, n), K_{\alpha}(\alpha \in P)$ subject to a certain set of $q$-analogues of Chevalley-Serre relations (this is a simple variation of the construction of Drinfeld and Jimbo). 
Recall that the braid group $\mathscr{B}_{W}$, associated to the given Cartan matrix, whose canonical generators one denotes by $T_{i}$ acts as a group of automorphisms of the algebra $U$ ([L]).

Introduce a conjugate-linear anti-automorphism $\kappa$ of $U$, viewed as an algebra over $\mathbb{C}$, by

$$
\kappa E_{i}=F_{i}, \kappa F_{i}=E_{\imath}, \kappa K_{i}=K_{i}^{-1}, \kappa q=q^{-1} .
$$

One knows that $\kappa$ commutes with the action of the braid group.

4.2. Fix a reduced expression $\omega_{0}=s_{i_{1}} \ldots s_{i_{N}}$ of the longest element of $W$, and let

$$
\beta_{1}=\alpha_{i_{1}}, \beta_{2}=s_{i_{1}}\left(\alpha_{i_{2}}\right), \ldots, \beta_{N}=s_{\imath_{1}} \ldots s_{i_{N-1}}\left(\alpha_{i_{N}}\right)
$$

be the corresponding convex ordering of $R^{+}$. Introduce the corresponding root vectors $(m=1, \ldots, N)([\mathrm{L}])$ :

$$
E_{\beta_{m}}=T_{\imath_{1}} \ldots T_{i_{m-1}} E_{i_{m}}, E_{-\beta_{m}}=T_{\imath_{1}} \ldots T_{i_{m-1}} F_{i_{m}}=\kappa E_{\beta}
$$

(they depend on the choice of the reduced expression).

For $k=\left(k_{1}, \ldots, k_{N}\right) \in \mathbb{Z}_{+}^{N}$ we let

$$
E^{k}=E_{\beta_{1}}^{k_{1}} \ldots E_{\beta_{N}}^{k_{N}}, F^{k}=\kappa E^{k} .
$$

Lemma 4.2. (a) [L] The elements $F^{k} K_{\alpha} E^{r}$, where $k, r \in \mathbb{Z}_{+}^{N}, \alpha \in P$, form a basis of $U$ over $\mathscr{A}$.

(b) $[L-S]$ For $i<j$ one has:

$$
E_{\beta_{j}} E_{\beta_{i}}-q^{-\left(\beta_{i} \mid \beta_{j}\right)} E_{\beta_{i}} E_{\beta_{\jmath}}=\sum_{k \in \mathbb{Z}_{+}^{N}} c_{k} E^{k}
$$

where $c_{k} \in \mathbb{C}\left[q, q^{-1}\right]$ and $c_{k} \neq 0$ only when $k=\left(k_{1}, \ldots, k_{N}\right)$ is such that $k_{s}=0$ for $s \leq i$ and $s \geq j$.

Consider a monomial

$$
M_{k, r, \alpha}:=F^{k} K_{\alpha} E^{r}
$$

where $k=\left(k_{1}, \ldots, k_{N}\right), r=\left(r_{1}, \ldots, r_{N}\right) \in \mathbb{Z}_{+}^{N}$ and $\alpha \in P$. Define its total height by

$$
d_{0}\left(M_{k, r, \alpha}\right)=\sum_{\imath}\left(k_{i}+r_{i}\right) \text { ht } \beta_{\imath}
$$

and its total degree by

$$
d\left(M_{k, r, \alpha}\right)=\left(k_{N}, k_{N-1}, \ldots, k_{1}, r_{1}, \ldots, r_{N}, d_{0}\left(M_{k, r, \alpha}\right)\right) \in \mathbb{Z}_{+}^{2 N+1}
$$

We shall view $\mathbb{Z}_{+}^{2 N+1}$ as a totally ordered semigroup with the lexicographical order $<$ such that $u_{1}<u_{2}<\ldots<u_{2 N+1}$, where $u_{i}=\left(\delta_{i, 1}, \ldots, \delta_{i, 2 N+1}\right)$.

Following [DC-K], introduce a $\mathbb{Z}_{+}^{2 N+1}$-filtration of the algebra $U$ by letting $U_{s}$ $\left(s \in \mathbb{Z}_{+}^{2 N+1}\right)$ be the span of the monomials $M_{k, r, \alpha}$ such that $d\left(M_{k, r, \alpha}\right) \leq s$. Lemma 4.2 implies 
Proposition 4.2 [DC-K]. The associated graded algebra $\mathrm{Gr} U$ of the $\mathbb{Z}_{+}^{2 N+1}$-filtered algebra $U$ is an algebra over to on generators $E_{\alpha}(\alpha \in R)$ and $K_{\beta}(\beta \in P)$ subject to the following relations:

$$
\begin{gathered}
K_{\alpha} K_{\beta}=K_{\alpha+\beta}, K_{0}=1 ; \\
K_{\alpha} E_{\beta}=q^{(\alpha \mid \beta)} E_{\beta} K_{\alpha} ; \\
E_{\alpha} E_{-\beta}=E_{-\beta} E_{\alpha} \text { if } \alpha, \beta \in R^{+} ; \\
E_{\alpha} E_{\beta}=q^{-(\alpha \mid \beta)} E_{\beta} E_{\alpha}, E_{-\alpha} E_{-\beta}=q^{-(\alpha \mid \beta)} E_{-\beta} E_{-\alpha}
\end{gathered}
$$

if $\alpha, \beta \in R^{+}$and $\alpha>\beta$ in our convex ordering of $R^{+}$.

Remark 4.2. (a) Considering the degree by total height $d_{0}$, we obtain a $\mathbb{Z}_{+}$-filtration of $U$; let $U^{(0)}=\bar{U}$ be the associated graded algebra. Letting $d_{1}\left(M_{k, r, \alpha}\right)=r_{N}$, we obtain a $\mathbb{Z}_{+}$-filtration of $U^{(0)}$; let $U^{(1)}=\bar{U}^{(0)}$ be the associated graded algebra. Letting $d_{2}\left(M_{k, r, \alpha}\right)=r_{N-1}$, we similarly obtain $U^{(2)}=\bar{U}^{(1)}$, etc. It is clear that at the last step we get the algebra Gr $U$ defined by (4.2.4-7):

$$
U^{(2 N)} \simeq \operatorname{Gr} U
$$

(b) The algebra Gr $U$ is a twisted polynomial algebra over to on generators $E_{\beta_{1}}, \ldots, E_{\beta_{N}}, E_{-\beta_{N}}, \ldots E_{-\beta_{1}}, K_{\omega_{1}}, \ldots, K_{\omega_{n}}$, corresponding to the skew-symmetric matrix $S$ defined by (3.3.1), with the elements $K_{\omega_{1}}, \ldots, K_{\omega_{n}}$ inverted.

We now specialize the variable $q$ to any non-zero complex number $\varepsilon$ subject to the further restriction $\varepsilon^{2 d_{\imath}} \neq 1$ and denote by $U_{\varepsilon}$ the corresponding specialized Hopf algebra.

From Lemma $4.2 \mathrm{a}$ we have that the monomials $M_{k, r, \alpha}$ form a basis of $U_{\varepsilon}$ over $\mathbb{C}$. Proposition 4.2 also holds for $U_{\varepsilon}$ with. $f$ replaced by $\mathbb{C}$ and $q$ replaced by $\varepsilon$. As in Remark 4.2 , we have a sequence of $\mathbb{Z}_{+}$-filtered algebras $U_{\varepsilon}^{(j)}$ over $\mathbb{C}$ $(j=0,1, \ldots, 2 N)$ such that $U_{\varepsilon}^{(j)}=\bar{U}_{\varepsilon}^{(\jmath-1)}$ and $U_{\varepsilon}^{(2 N)}$ is the algebra described by Remark 4.2 b with. $A$, replaced by $\mathbb{C}$.

4.3. Recall the following general construction (see, e.g. [DC-K-P]). Given $\varepsilon \in \mathbb{C}^{\times}$, let $\varphi: U \rightarrow U_{\varepsilon}=U /(q-\varepsilon)$ be the specialization at $q=\varepsilon$. Let $Z_{\varepsilon}$ be the center of $U_{\varepsilon}$ and let $D_{\varepsilon}=\varphi^{-1}\left(Z_{\varepsilon}\right)$. Then for any element $a \in D_{\varepsilon}$ we can define the associated Poisson derivation $P_{a}$ of $U_{\varepsilon}$ by the formula

$$
P_{a}(u)=c \frac{a \varphi^{-1}(u)-\varphi^{-1}(u) a}{q-\varepsilon} \bmod (q-\varepsilon),
$$

where $c$ is a normalization factor. It is clear that $P_{a}$ is a well-defined derivation of $U_{\varepsilon}$ (hence it maps $Z_{\varepsilon}$ into itself), satisfying

$$
P_{a b}(u)=P_{a}(u) \varphi(b)+\varphi(a) P_{b}(u), a, b \in D_{\varepsilon} .
$$

In particular, we obtain a Poisson bracket on $Z_{\varepsilon}$ :

$$
\{a, b\}:=P_{\varphi^{-1}(a)}(b)=-P_{\varphi^{-1}(b)}(a) .
$$

4.4. Let $\ell$ be an odd integer greater than 1 and relatively prime to all the $d_{i}$, and let $\varepsilon$ be a primitive $\ell^{\text {th }}$ root of 1 . Let as before $Z_{\varepsilon}$ denote the center of the algebra $U_{\varepsilon}$. One knows [DC-K] that the elements $E_{\alpha}^{\ell}(\alpha \in R)$, and $K_{\beta}^{\ell}(\beta \in P)$ lie in $Z_{\varepsilon}$; we denote 
by $Z_{0}$ the subalgebra of $Z_{\varepsilon}$ generated by all these elements. The subalgebras $Z_{\varepsilon}$ and $Z_{0}$ are $\mathscr{\beta}_{W}$-invariant [DC-K] and possess a natural Poisson structure (cf. Sect. 4.3):

$$
\{a, b\}=\frac{\varphi^{-1}(a) \varphi^{-1}(b)-\varphi^{-1}(b) \varphi^{-1}(a)}{2 \ell^{2}(q-\varepsilon)} \bmod (q-\varepsilon), a, b \in Z_{\varepsilon} .
$$

Moreover $Z_{0}$ is a Hopf subalgebra and in fact it is the coordinate ring of a Poisson algebraic group $H$ described below [DC-K-P].

Let $G$ be the simply connected algebraic group over $\mathbb{C}$ with Lie algebra $\mathfrak{g}$ associated to our Cartan matrix. Let $T$ be the maximal torus of $G$ with Lie algebra $\mathfrak{h}:=\sum_{i} \mathbb{C} h_{i}$. Let $\mathfrak{n}_{-}\left(\right.$resp. $\left.\mathfrak{n}_{+}\right)$be the subalgebra of $\mathfrak{g}$ generated by the $f_{i}$ (resp. $e_{i}$ ) and let $U_{-}$ and $U_{+}$be the unipotent subgroups of $G$ whose Lie algebras are $\mathfrak{n}_{-}$and $\mathfrak{n}_{+}$. Let $B_{-}:=T U_{-}$and $B_{+}:=T U_{+}$be the corresponding Borel subgroups. Recall that the braid group $\mathscr{P}_{W}$ acts on $\mathfrak{g}$ by letting [T]:

$$
T_{i}=\left(\exp \operatorname{ad} f_{i}\right)\left(\exp \operatorname{ad} e_{i}\right)\left(\exp \operatorname{ad} f_{i}\right)
$$

For each $\alpha \in R^{+}$we define root vectors $e_{\alpha}$ and $e_{-\alpha}$ by formulas analogous to (4.2.1).

The group $H$ is the subgroup of $B_{-} \times B_{+}$formed by the elements $(x, y)=$ $\left(t u_{-}, t^{-1} u_{+}\right)$. The restriction to $H$ of the natural multiplication map $B_{-} \times B_{+} \rightarrow G$ given by $(x, y) \rightarrow x^{-1} y$ is an etale covering of degree $2^{n}$ :

$$
\pi: H \rightarrow U_{-} T U_{+}:=G^{0} \subset G
$$

where $G^{0}$ is a Zariski open subset of $G$, called the big cell. We need to recall the explicit isomorphism between $H$ and $\operatorname{Spec} Z_{0}$ [DC-K-P].

We let for $\alpha \in R^{+}$:

$$
d_{\alpha}=\frac{1}{2}(\alpha \mid \alpha), c_{\alpha}=\left(\varepsilon^{d_{\alpha}}-\varepsilon^{-d_{\alpha}}\right)^{\ell},
$$

and introduce the following elements of $Z_{0}$ :

$$
z_{\beta}=K_{\beta}^{\ell}(\beta \in P) ; x_{-\alpha}=c_{\alpha} E_{-\alpha}^{\ell}, x_{\alpha}=-c_{\alpha} E_{\alpha}^{\ell} z_{-\alpha}\left(\alpha \in R^{+}\right) .
$$

Let $Z_{0}^{0}=\sum_{\beta \in P} \mathbb{C} z_{\beta}$ and let $Z_{0}^{+}$(resp. $Z_{0}^{-}$) be the subalgebra of $Z_{0}$ generated by the elements $x_{\alpha}$ (resp. $x_{-\alpha}$ ), $\alpha \in R^{+}$. Then [DC-K] the algebra $Z_{0}^{+}$(resp. $Z_{0}^{-}$) is a polynomial algebra on indeterminants $x_{\alpha}$ (resp. $x_{-\alpha}$ ) and multiplication defines an algebra isomorphism

$$
Z_{0} \simeq Z_{0}^{-} \otimes Z_{0}^{0} \otimes Z_{0}^{+}
$$

Since $\mathbb{C}[T]=P$, we have a canonical isomorphism of $T$ with $\operatorname{Spec} Z_{0}^{0}$, and we shall identify them.

Following [DC-K-P] we construct maps

$$
\pi^{-}: \operatorname{Spec} Z_{0}^{-} \rightarrow U_{-}, \pi^{+}: \operatorname{Spec} Z_{0}^{+} \rightarrow U_{+}
$$

and then construct an isomorphism $\tilde{\pi}: \operatorname{Spec} Z_{0}=\operatorname{Spec} Z_{0}^{-} \times T \times \operatorname{Spec} Z_{0}^{+} \rightarrow H$ by letting

$$
\tilde{\pi}(a, t, b):=\left(t^{-1} \pi^{-}(a), t \pi^{+}(b)\right) .
$$

Here the map $\pi^{-}$is given by the following element of the group $U_{-}\left(Z_{0}^{-}\right) \subset G\left(Z_{0}\right)$

$$
\left(\exp x_{-\beta_{N}} e_{-\beta_{N}}\right) \ldots\left(\exp x_{-\beta_{1}} e_{-\beta_{1}}\right)
$$


and the map $\pi^{+}$by the following element of the group $U_{+}\left(Z_{0}^{+}\right) \subset G\left(Z_{0}\right)$ :

$$
\left(\exp T_{0}\left(x_{-\beta_{N}}\right) T_{0}\left(e_{-\beta_{N}}\right)\right) \ldots\left(\exp T_{0}\left(x_{-\beta_{1}}\right) T_{0}\left(e_{-\beta_{1}}\right)\right)
$$

where $T_{0}=T_{i_{1}} \ldots T_{i_{N}}$. We identify $\operatorname{Spec} Z_{0}$ and $H$ using the isomorphism $\tilde{\pi}$, hence we have an etale covering $\pi: \operatorname{Spec} Z_{0} \rightarrow G^{0} \subset G$ of degree $2^{n}$.

Remark 4.4. Since also $T_{0}=T_{i_{N}} \ldots T_{i_{1}}$, we see that $-T_{0} \beta_{N}=\alpha_{i_{N}},-T_{0} \beta_{N-1}=$ $T_{i_{N}} \alpha_{i_{N-1}}, \ldots,-T_{0} \beta_{1}=T_{i_{N}} \ldots T_{i_{2}} \alpha_{i_{1}}$, which is the convex ordering of $R^{+}$ associated to the "reverse" reduced expression $\omega_{0}=s_{i_{N}} \ldots s_{i_{1}}$. As in (4.2.1) we have the corresponding root vectors, but they may be different from those in (4.2.1).

4.5. It is clear that $U_{\varepsilon}$ is a finite module over $Z_{0}$. In [DC-K] it is shown that it is a maximal order in a division algebra of degree $d=l^{N}, N=\left|R^{+}\right|$. Thus we can apply to this algebra the general theory described in Sect. 1.

Let $V:=\operatorname{Spec} Z_{\varepsilon}$. The points of $V$ parametrize, according to Sect. 1 , the semisimple $d$-dimensional representations of $U_{\varepsilon}$. In ([DC-K-P]) we have described $V$ as a canonical ramified covering of $H$ which has also a Poisson structure compatible with the covering map.

A basic symmetry of our picture is associated to the Poisson structure of $H$. In fact we have a canonical (infinite dimensional) group $\tilde{G}$ of analytic automorphisms of the algebra $U_{\varepsilon}$ generated by 1-parameter groups $\exp t P_{E_{\imath}^{\ell}}$ and $\exp t P_{F_{\imath} \ell}(i=1, \ldots, n)$ which covers a group of Hamiltonian flows on $H$ [DC-K]. In particular:

(a) The group $\tilde{G}$ acts on $V$ and on $H$ and its orbits coincide with the symplectic leaves.

(b) The semisimple representations of $U_{\varepsilon}$ parametrized by the points of a given symplectic leaf in $V$ are all of the same "type" (i.e. are equivalent up to a twist by an automorphism of $U_{\varepsilon}$ ).

We shall describe now the semisimple $d$-dimensional representation of $U_{\varepsilon}$ corresponding to a given point of $\operatorname{Spec} Z_{\varepsilon}$. Recall [DC-K] that given a homomorphism $\lambda: U_{\varepsilon}^{0} \rightarrow \mathbb{C}$ and a homomorphism $\nu: Z_{0}^{-} \rightarrow \mathbb{C}$ we construct the associated left $U_{\varepsilon}$-module $\bar{M}_{\varepsilon}(\lambda, \nu)$, called the triangular Verma module, as the quotient of $U_{\varepsilon}$ by the left ideal generated by the elements $E_{\alpha}, K_{\gamma}-\lambda\left(K_{\gamma}\right), x_{-\alpha}-\nu\left(x_{-\alpha}\right)$, where $\gamma \in P, \alpha \in R^{+}$. This is a $d$-dimensional indecomposable representation of $U_{\varepsilon}$. Hence all irreducible factors $\rho_{1}, \ldots, \rho_{s}$ of $\bar{M}_{\varepsilon}(\lambda, \nu)$ have the same central character $\chi \in \operatorname{Spec} Z_{\varepsilon}$.

Proposition 4.5. (a) The representation $\bigoplus_{i=1}^{s} \rho_{\imath}$ is the semisimple representation of $U_{\varepsilon}$ corresponding to $\chi \in \operatorname{Spec} Z_{\varepsilon}$.

(b) Given $\chi \in \operatorname{Spec} Z_{\varepsilon}$, choose $g \in \tilde{G}$ such that $\chi_{1}=g(\chi)$ has the property that $\chi_{1}\left(x_{\alpha}\right)=0$ for all $\alpha \in R^{+}$(see Proposition 4.6 below), and let $\nu=\left.\chi_{1}\right|_{z_{0}^{-}}$. Then there exists a homomorphism $\lambda: U_{\varepsilon}^{0} \rightarrow \mathbb{C}$ such that the central character of the module $\bar{M}_{\varepsilon}(\lambda, \nu)$ is equal to $\chi_{1}$; let $\rho_{1}, \ldots, \rho_{s}$ be all its irreducible factors. Then $\bigoplus_{i=1}^{s} \rho_{i}^{g^{-1}}$ is the semisimple representation of $U_{\varepsilon}$ corresponding to $\chi$.

(c) Triangular Verma modules having the same central character have the same irreducible factors.

Proof. (b) and (c) follows from (a). Due to the discussion in Sect. 1.1, it suffices to show that the triangular Verma modules $\bar{M}_{\varepsilon}(\lambda, \nu)$ are compatible with the trace 
map. It is obviously the case if $\bar{M}_{\varepsilon}(\lambda, \nu)$ is irreducible. But the module $\bar{M}_{\varepsilon}(\lambda, \nu)$ is irreducible provided that $\lambda\left(z_{\beta}\right) \neq \pm 1$ for all $\beta \in R^{+}$[DC-K, Corollary 3.2]. Hence, by continuity, all the modules $\bar{M}_{\varepsilon}(\lambda, \nu)$ are compatible with the trace map.

4.6. Let $\lambda \in P_{+}$be a dominant weight and let $\rho_{\lambda}$ be the finite-dimensional irreducible representation of the group $G$ in a vector space $V^{\lambda}$. Then we have a $\operatorname{map} \pi_{\lambda}=\rho_{\lambda} \circ \pi: \operatorname{Spec} Z_{0} \rightarrow G L\left(V^{\lambda}\right)$. Let

$$
\phi_{\lambda}(u)=\operatorname{tr}_{V^{\lambda}} \pi_{\lambda}(u), u \in \operatorname{Spec} Z_{0} .
$$

This is a polynomial function on $\operatorname{Spec} Z_{0}$.

Proposition 4.6 [DC-K-P]. (a) $\pi^{-1}(\operatorname{Center}(G))$ is the fixed point set $F$ of $\tilde{G}$ in Spec $Z_{0}$ (it is a finite set of cardinality $2^{n}|\operatorname{Center}(G)|$ ).

(b) Let $\odot$ be a non-central conjugacy class of $G$. Then $\pi^{-1}\left(\odot \cap G^{0}\right)$ is a $\tilde{G}$-orbit and all $\tilde{G}$-orbits in Spec $Z_{0} \backslash F$ are thus obtained.

(c) The elements $\phi_{\lambda}\left(\lambda \in P_{+}\right)$form a basis over $\mathbb{C}$ of the subalgebra $Z_{0}^{\tilde{G}}$ of $\tilde{G}_{\text {- }}$ invariants in $Z_{0}$.

(d) The algebra $Z_{0}^{\tilde{G}}$ is a polynomial algebra over $\mathbb{C}$ on generators $\phi_{\omega_{1}}, \ldots, \phi_{\omega_{n}}$.

Remark 4.6 (cf. Remark 3.3.) Let $\left(a_{i j}\right)$ be the Cartan matrix of type $G_{2}$ and let $\ell=3 \ell^{\prime}$, where $\ell^{\prime}$ is an odd integer greater than 1 . Then $E_{\alpha}^{\ell^{\prime}}$ is a central element of $U_{\varepsilon}$ if $\alpha$ is a long root. Proposition 4.6 holds in this case as well if we replace $E_{\alpha}^{\ell}$ by $E_{\alpha}^{\ell^{\prime}}$ in all constructions.

We return now to the $\mathbb{Z}_{+}^{2 N+1}$-filtration of the algebra $U_{\varepsilon}$ obtained by the specialization of that of $U_{\mathscr{C}}$ (see Sect. 4.2). Recall that the monomials $M_{k, r, \alpha}$ (given by (4.2.3)) form a basis of $U_{\varepsilon}$ over $\mathbb{C}$ (see Lemma 4.2a). For an element $\phi$ of $U_{\varepsilon}$ we denote by $\bar{\phi}$ the monomial of maximal degree in the above filtration that occurs in $\phi$ with a non-zero coefficient.

Fix a reduced expression (3.2.1) of $\omega_{0}$. Fix a fundamental weight $\omega$, let $\alpha$ be the corresponding simple root and let

$$
I_{\alpha}=\left\{\beta_{k_{1}}, \ldots, \beta_{k_{r}}\right\}
$$

be the corresponding subset defined by (3.2.2).

Lemma 4.6. Let $\phi_{\omega}$ be the element of $Z_{0}$ defined by (4.6.1) for $\lambda=\omega$. Then

$$
\bar{\phi}_{\omega}=z_{\omega}^{2} x_{-\beta_{k_{r}}} \ldots x_{-\beta_{k_{1}}} x_{\beta_{k_{1}}} \ldots x_{\beta_{k_{r}}} .
$$

Proof. Fix a basis $\left\{v_{j}\right\}, j=1, \ldots, t=\operatorname{dim} V^{\omega}$, of $V^{\omega}$ such that $v_{j}$ is a weight vector of weight $\mu_{j}$ and $i<j$ if $\mu_{i}>\mu_{j}$, so that $v_{t}$ has the highest weight $\omega$ and $v_{1}$ has the lowest weight $-{ }^{t} \omega$. Let $\left\{v_{j}^{*}\right\}$ be the dual basis of $\left(V^{\omega}\right)^{*}$ and consider the matrix coefficients

$$
S_{i j}(g)=\left\langle\pi_{\omega}(g) v_{j}, v_{j}^{*}\right\rangle, g \in G
$$

We define the height of a monomial in $Z_{0}$ by $H\left(z_{\alpha} \prod_{i} x_{-\beta_{i}}^{k_{2}} x_{\beta_{2}}^{r_{2}}\right)=\sum_{i} \beta_{i}\left(r_{i}+k_{i}\right)$, and we write $H(z) \leq \lambda$ for $z \in Z_{0}$ and $\lambda \in Q$ if the heights of all monomials in $z$ are $\leq \lambda$. 
Note the following properties of matrix coefficients:

$$
\begin{gathered}
S_{i j} \circ \pi_{+}=\delta_{i j} \text { if } i \leq j ; H\left(S_{i j} \circ \pi_{+}\right) \leq \mu_{i}-\mu_{j} ; \\
S_{i j} \circ \pi_{-}=\delta_{i j} \text { if } i \geq j ; H\left(S_{i j} \circ \pi_{-}\right) \leq \mu_{j}-\mu_{\imath} ; \\
S_{i j} \circ \pi_{0}=\delta_{\imath j} z_{\mu_{\imath}}^{2} ; H\left(S_{\imath \jmath} \circ \pi_{0}\right)=0 .
\end{gathered}
$$

Since

$$
S_{\jmath \jmath} \circ \pi=\sum_{j \leq i \leq t}\left(S_{j i} \circ \pi_{-}\right)\left(S_{i i} \circ \pi_{0}\right)\left(S_{i j} \circ \pi_{+}\right),
$$

we deduce from (4.6.2-4) that

$$
H\left(S_{j \jmath} \circ \pi\right) \leq 2\left(\omega-\mu_{j}\right) .
$$

Since

$$
\phi_{\omega}=\sum_{j} S_{j \jmath} \circ \pi
$$

it follows from (4.6.5) that the highest possible height of a monomial in $S_{\jmath j} \circ \pi$ occurs for $j=1$ and it is equal to $2\left(\omega+{ }^{t} \omega\right)$. Moreover, this monomial may occur only in the summand $\left(S_{1 t} \circ \pi_{-}\right)\left(S_{t t} \circ \pi_{0}\right)\left(S_{t 1} \circ \pi_{+}\right)$of $S_{11} \circ \pi$.

Thus, it remains to show that the highest degree monomial (with respect to our $\mathbb{Z}_{+}^{2 N+1}$-filtration) in $\left(S_{1 t} \circ \pi_{-}\right)\left(S_{t t} \circ \pi_{0}\right)\left(S_{t 1} \circ \pi_{+}\right)$is the monomial $\bar{\phi}_{\omega}$. But $S_{t t} \circ \pi_{0}=z_{\omega}^{2}$, hence it remains to show that the highest degree monomial in $S_{1 t} \circ \pi_{-}\left(\right.$resp. $\left.S_{t 1} \circ \pi_{+}\right)$is $x_{-\beta_{k_{1}}} \ldots x_{-\beta_{k_{r}}}$ (resp. $x_{\beta_{k_{1}}} \ldots x_{\beta_{k_{r}}}$ ). (Recall that, by Lemma $3.2 \mathrm{~d}^{\prime}, \omega+{ }^{t} \omega=\sum_{i=1}^{r} \beta_{k_{i}}$.)

But this follows immediately, recalling formulas (4.3.1) and (4.3.2) from Lemma 3.2 $\mathrm{b}^{\prime}$ and $\mathrm{c}^{\prime}$ (resp. $\mathrm{b}$ and $\mathrm{c}$ )

\section{The Main Theorems}

5.1. As before, we assume that $\ell$ is an odd integer greater than 1 and relatively prime to all the $d_{\imath}$. Let $\varepsilon$ be a primitive $\ell^{\text {th }}$ root of 1 and let $d$ be the degree of the algebra $U_{\varepsilon}$. We have already recalled in Sect. 4.5 that $d=\ell^{N}$.

We are in a position now to prove the main result of the paper.

Theorem 5.1 Let $V$ be a irreducible $U_{\varepsilon}$-module and let $\left(\mathcal{G}\right.$ be the $\tilde{G}$-orbit of $\chi_{0}(V)$ in Spec $Z_{0}$. If $\odot$ has maximal dimension $(=2 N)$, then $V$ has maximal dimension $(=d)$.

Proof. Let $\Omega^{0}=\Omega_{U_{\varepsilon}}^{0}=\left\{u \in\right.$ Spec $Z_{0} \mid$ all representations from $\chi_{0}^{-1}(u)$ have dimension $d\}$, as introduced in Sect. 1.2. We are claiming that, given a $\tilde{G}$-orbit (Q) of maximal dimension, $\odot \subset \Omega^{0}$. By symmetry (cf. Sect. 4.4) it suffices to see that the Zariski closure $\overline{\mathscr{Q}}$ of $\mathscr{Q}$ has nonempty intersection with $\Omega^{0}$. We wish to apply to our situation Lemma 1.5 with $I$ the ideal of $\overline{\mathcal{Q}}$. For this we need first of all, to show that each of the algebras $U_{\varepsilon}^{(j)}$ introduced in Remark 4.2 has degree $\ell^{N}$. Since the degree can only decrease in each step, it is enough to show that $d$ is the degree of $U_{\varepsilon}^{(2 N)}$. In this case we apply Proposition 2.2c and Lemma 3.3b. By an easy induction we are then reduced to show that, if $\bar{I}$ is the associated graded ideal of $I$ in $U_{\varepsilon}^{(2 N)}$, and $\mathscr{Q}_{1}$ is its set of zeroes, then

$$
\mathscr{Q}_{1} \cap \Omega_{U_{\varepsilon}^{(2 N)}}^{0} \neq \emptyset .
$$


We will describe $\bar{I}$ using the method of Proposition 1.4. It is well known that the Zariski closure of $\pi(\odot)$, being the Zariski closure of the conjugacy class of maximal dimension in $G$, is given by the equations (for some $c_{i} \in \mathbb{C}$ ):

$$
\operatorname{tr}_{V^{\omega_{\imath}}} g=c_{i}, i=1, \ldots, u, g \in \overline{\pi(\mathscr{O})} \text {. }
$$

It follows from Proposition $4.6 \mathrm{~b}$ and $\mathrm{d}$ that the Zariski closure of $\mathcal{O}$ in $\operatorname{Spec} Z_{0}$ is given by the equations

$$
\phi_{\omega_{i}}=c_{i}, i=1, \ldots, n \text {. }
$$

Consider the elements $\bar{\phi}_{\omega_{\imath}}$, images of $\phi_{\omega_{\imath}}$ in the final graded algebra $U_{\varepsilon}^{(2 N)}$. We want to show that these elements form a regular sequence of $Z_{0}^{(2 N)} \subset U_{\varepsilon}^{(2 N)}$ so that they generate $\bar{I}$ (cf. Proposition 1.4). The elements $\bar{\phi}_{\omega_{2}}$ have been computed in Lemma 4.6 where we have seen that they are monomials in disjoint sets of indeterminates. Hence they form a regular sequence by trivial reasons. In order to complete the proof we have to show that the set $\mathscr{O}_{1}$ of solutions of the system of equations

$$
\bar{\phi}_{\omega_{i}}=0, i=1, \ldots, n \text {, }
$$

intersects nontrivially the set $\Omega_{U^{(2 N)}}^{0}$.

The variety given by the equations (5.1.3) is a union of subvarieties given as follows: we choose from each monomial $\bar{\phi}_{\omega_{i}}$ a factor $x_{ \pm \beta}$ and letting them 0 we define a component of the variety under study. It is enough to prove that each one of these subvarieties intersects the open set $\Omega_{U^{(2 N)}}^{0}$ non-trivially. But $U_{\varepsilon}^{(2 N)}$ is essentially a twisted polynomial algebra (see Remark $4.2 \mathrm{~b}$ ). Thus, according to Proposition $2.2 \mathrm{~d}$ and Lemma 2.2, the statement follows from Lemma 3.3c, completing the proof.

Remark 5.1 (cf. Remarks 3.3 and 4.4). Let $\ell$ be an odd integer greater than $d_{i}$ for all $i$. For each $\alpha \in R^{+}$let $\ell_{\alpha}=\ell /\left(\ell, d_{\alpha}\right)$. (Note that $\ell_{\alpha}=\ell$ in all cases except for long $\alpha$ of type $G_{2}$ and $\ell$ divisible by 3 .) Then

$$
d=\prod_{\alpha \in R^{+}} \ell_{\alpha} .
$$

Here is a simple proof of this formula. Since degree $U_{\varepsilon} \geq \operatorname{degree} U_{\varepsilon}^{(2 N)}$, we deduce from Proposition 3.3 and Proposition 4.2c (and Remark 3.3) that $d \geq \prod_{\alpha} \ell_{\alpha}$. Using the triangulizability of an arbitrary irreducible representation of $U_{\varepsilon}$ (which follows from Proposition 4.6b), we obtain the reverse inequality.

Theorem 5.1 holds for these $\ell$ as well.

5.2. In this last section we denote by $U$ the algebra $U \otimes_{\ell} \mathbb{C}(q)$, let $U^{(2 N)}=\mathrm{Gr} U$ etc. We discuss in some detail the center $\bar{Z}$ of $\operatorname{Gr} U$ (resp. $\bar{Z}_{\varepsilon}$ of $\operatorname{Gr} U_{\varepsilon}$ ) having in mind some possible applications to a more detailed analysis of the geometry of the degeneration. Recall that this is an algebra over $\mathbb{C}(q)$ (resp. $\mathbb{C}$ ) on generators $E_{\alpha}$ $(\alpha \in R)$ and $K_{\beta}(\beta \in P$ ) with defining relations (4.2.4-7) (resp. where $q=\varepsilon$ ). Let $Z$ denote the center of $U$. From Proposition 2.2a and Lemma 3.3 we derive

Proposition 5.2. (a) The algebra $\bar{Z}$ is a polynomial algebra over $\mathbb{C}(q)$ on generators

$$
u_{\imath}:=K_{\omega_{\imath}-{ }^{t} \omega_{i}} \prod_{t \in I_{\alpha_{\imath}}} E_{\beta_{t}} E_{-\beta_{t}} \quad(i=1, \ldots, n) .
$$


(b) The algebra $\bar{Z}_{\varepsilon}$ is an algebra over $\mathbb{C}$ on generators $\bar{x}_{\alpha}:=E_{\alpha}^{\ell}(\alpha \in R), \bar{z}_{\beta}:=K_{\beta}^{\ell}$ $(\beta \in P)$ and $u_{i}(i=1, \ldots, n)$ with the following defining relations:

$$
\begin{aligned}
\bar{z}_{\alpha} \bar{z}_{\beta} & =\bar{z}_{\alpha+\beta}, \quad \bar{z}_{0}=1 \\
u_{i}^{\ell} & =\bar{z}_{\omega_{i}-{ }^{t} \omega_{i}} \prod_{t \in I_{\alpha_{\imath}}} \bar{x}_{\beta_{t}} \bar{x}_{-\beta_{t}} \quad(i=1, \ldots, n) .
\end{aligned}
$$

Theorem 5.2. (a) $\bar{Z}=\operatorname{Gr} Z$.

(b) $\bar{Z}_{\varepsilon}=\operatorname{Gr} Z_{\varepsilon}$.

Proof. It is clear that $\bar{Z} \supset \mathrm{Gr} Z$ and $\bar{Z}_{\varepsilon} \supset \mathrm{Gr} Z_{\varepsilon}$. In order to prove the reverse inclusion recall the construction of $Z$ from [DC-K]. Let $U^{0}$ (resp. $U^{+}$and $U^{-}$) be the subalgebra of $U$ generated by all the $K_{\alpha}\left(\alpha \in P\right.$ ) (resp. all the $E_{\imath}$ and all the $F_{i}$ ). Recall that $W$ acts on $U^{0}$ by $w K_{\alpha}=K_{w(\alpha)}$. Let $U^{0 W}$ denote the subalgebra of invariants. Given $\varphi=\varphi\left(K_{\omega_{1}}, \ldots, K_{\omega_{n}}\right) \in U^{0 W}$, there exists a unique element in $Z$ of the form:

$$
p_{\varphi}=\varphi\left(q^{2\left(\rho \mid \omega_{1}\right)} K_{\omega_{1}}^{2}, \ldots, q^{2\left(\rho \mid \omega_{n}\right)} K_{\omega_{n}}^{2}\right)+\sum_{\beta \in Q_{+} \backslash\{0\}} S_{\beta}^{\varphi}
$$

where $S_{\beta}^{\varphi} \in U_{-\beta}^{-} U^{0} U_{\beta}^{+}$and $U_{\beta}^{ \pm}=\left\{u \in U^{ \pm} \mid K_{\alpha} u K_{-\alpha}=q^{(\alpha \mid \beta)} u\right\}$. Furthermore, the map $h: p_{\varphi} \mapsto \varphi$ defines an isomorphism $Z \stackrel{\sim}{\longrightarrow} U^{0 W}$.

The algebra $U^{0}$ is canonically isomorphic to the coordinate ring of the torus $T$ of $G$. Given $\lambda \in P_{+}$define an element $\chi_{\lambda} \in U^{0}$ by $\chi_{\lambda}(t)=\operatorname{tr}_{V^{\lambda}} \pi_{\lambda}(t), t \in T$. Of course, $\chi_{\lambda} \in U^{0 W}$ and we let $p_{\lambda}=p_{\chi_{\lambda}} \in Z$. The elements $p_{\omega_{1}}, \ldots, p_{\omega_{n}}$ generate $Z$. Let $\bar{p}_{\varphi}$ denote the monomial of highest degree in $p_{\varphi}$ with respect to the $\mathbb{Z}_{+}^{2 N+1}$-filtration of $U$ (defined in Sect. 4.2). In order to prove (a), it suffices to show that

$$
\bar{p}_{\omega_{i}}=u_{\imath} .
$$

Since the elements $p_{\omega_{i}}$ are defined at $q=\varepsilon$ and together with $Z_{0}$ they generate $Z_{\varepsilon}$ [DC-K-P], (5.2.2) implies (b) as well.

In order to prove (5.2.2) we use another approach to $Z$, developed in [R] and [Ta]. First, one introduces the unique bilinear form $(.,):. U^{0} U^{+} \times U^{0} U^{-} \rightarrow \mathbb{F}:=$ $\mathbb{C}\left(q^{1 / 2 \operatorname{det}\left(a_{\imath j}\right)}\right)$ satisfying the following relations:

$$
\begin{gathered}
\left(x, y_{1} y_{2}\right)=\left(\Delta(x), y_{1} \otimes y_{2}\right), \quad x \in U^{0} U^{+}, \quad y_{i} \in U^{0} U^{-}, \\
\left(x_{1} x_{2}, y\right)=\left(x_{2} \otimes x_{1}, \Delta(y)\right), \quad x_{1}, x_{2} \in U^{0} U^{+}, \quad y \in U^{0} U^{-}, \\
\left(K_{\alpha}, K_{\beta}\right)=q^{-(\alpha \mid \beta)}, \alpha, \beta \in P, \\
\left(E_{\imath}, K_{\alpha}\right)=\left(K_{\alpha}, F_{i}\right)=0, \quad \alpha \in P, \quad i=1, \ldots, n, \\
\left(E_{\imath}, F_{j}\right)=\delta_{i j} /\left(q^{-d_{\imath}}-q^{d_{i}}\right), \quad i, j=1, \ldots, n .
\end{gathered}
$$

Then one has

$$
\begin{gathered}
\left(U_{\alpha}^{+}, U_{-\beta}^{-}\right)=0 \text { if } \alpha \neq \beta, \\
(., .) \text { on } U_{\alpha}^{+} \times U_{-\alpha}^{-} \text {is non-degenerate. }
\end{gathered}
$$


Furthermore, using that $U=U^{-} U^{0} U^{+}=U^{+} U^{0} U^{-}$, one extends this bilinear form to the whole algebra $U$. by the formula:

$$
\begin{gathered}
\left(x_{1} K_{\alpha} S\left(y_{1}\right), y_{2} K_{\beta} S\left(x_{2}\right)\right)=\left(x_{1}, y_{2}\right)\left(x_{2}, y_{1}\right) q^{-(\alpha \mid \beta)} \\
x_{i} \in U^{+}, y_{\imath} \in U^{-}, \alpha, \beta \in P .
\end{gathered}
$$

This bilinear form defines a linear map

$$
j: U \rightarrow U^{*}\left(=\operatorname{Hom}_{\mathbb{F}}(U, \mathbb{F})\right) \text { by } j(a)(u)=(u, a) .
$$

The basic fact of $[\mathrm{R}]$ and [Ta] is that, considering the irreducible representation $\pi_{\lambda, q}$ of $U$ in the vector space $V_{q}^{\lambda}$ over $\mathbb{C}(q)$ which is a deformation of $\left(V^{\lambda}, \pi_{\lambda}\right)$, we obtain:

$$
j\left(p_{\lambda}\right)(u)=\operatorname{tr}_{V_{q}^{\lambda}} \pi_{\lambda, q}\left(K_{-2 \rho} u\right), u \in U
$$

Let us now remark that any weight of $V^{\lambda}$ has the form $\lambda-\beta$, where $\beta \in Q_{+}$and $\beta \leq \lambda+{ }^{t} \lambda$. Hence it follows from (5.2.11) and the definition of the bilinear form on $U$ that in (5.2.1) we have:

$$
S_{\beta}^{\chi_{\lambda}} \neq 0 \Rightarrow \beta \leq \lambda+{ }^{t} \lambda
$$

Furthermore, let $\chi_{\lambda}{ }^{\prime}=\sum_{w \in W / W_{\lambda}} K_{w(\lambda)}\left(\lambda \in P_{+}\right)$and let $p_{\lambda}^{\prime}=p_{\chi_{\lambda}^{\prime}}$. Since

$$
\chi_{\lambda}^{\prime}=\chi_{\lambda}+\sum_{\substack{\beta: 0<\beta \leq \lambda+{ }^{t} \lambda, \lambda-\beta \in P_{+}}} c_{\lambda-\beta} \chi_{\lambda-\beta}, \text { where } c_{\lambda-\beta} \in \mathbb{C},
$$

we deduce from (5.2.12) that

$$
S_{\beta}^{\chi_{\lambda}^{\prime}} \neq 0 \Rightarrow \beta \leq \lambda+{ }^{t} \lambda
$$

and we see that

$$
\bar{p}_{\lambda}=\overline{p_{\lambda}^{\prime}}
$$

On the other hand, choosing $\ell$ relatively prime to all the $d_{i}$ we have from Proposition 4.6 in $U_{\varepsilon}$ for a fundamental weight $\omega$ :

$$
\overline{p_{\ell \omega}^{\prime}}=K_{\omega-t_{\omega}}^{\ell} E_{-\beta_{k_{1}}}^{\ell} \ldots E_{-\beta_{k_{r}}}^{\ell} E_{\beta_{1}}^{\ell} \ldots E_{\beta_{k_{r}}}^{\ell} .
$$

By (5.2.13) we have in $U_{\varepsilon}$ :

$$
\overline{p_{\lambda}^{\prime \ell}}=\left(\overline{p_{\lambda}^{\prime}}\right)^{\ell}=\overline{p_{\ell \lambda}^{\prime}}, \lambda \in P_{+} .
$$

Choosing $\ell$ big enough, we may assume that the highest monomials of $p_{\omega}$ and $p_{\omega}^{\prime}$ do not vanish at $q=\varepsilon$, hence coincide according to (5.2.14). Therefore comparing (5.2.15) and (5.2.16) gives (5.2.2) which completes the proof of the theorem.

Corollary 5.2. Let $Z_{\varepsilon}^{(j)}$ be the center of the algebra $U_{\varepsilon}^{(j)}$. Then $Z^{(j+1)}=\overline{Z^{(j)}}$. 


\section{References}

[A] Artin, M.: On Azumaya algebras and finite dimensional representations of rings. J. Algebra 11 532-563 (1969)

[B] Bourbaki, N.: Algebre. Ch X, Paris: Algebre homologique 1980

[DC-K] De Concini, C.; Kac, V.G.: Representations of quantum groups at roots of 1. In: Progress in Math., 92. Basel: Birkhäuser 1990, pp. 471-506

[DC-K-P] De Concini, C.; Kac, V.G.; Procesi, C.: Quantum coadjoint action. J. AMS 5 151-190 (1992)

[L] Lusztig, G.: Quantum groups at roots of 1. Geom. Ded. 35 89-114 (1990)

[LS] Levendorskii, S.Z.; Soibelman, Ya. S.: Algebras of functions on compact quantum groups, Schubert cells and quantum tori. Commun. Math. Phys. 139 141-170 (1991)

[P1] Procesi, C.: Rings with polynomial identities. Pure Appl. Math. 17, M. Dekker 1973

[P2] Procesi, C.: Finite dimensional representations of algebras. Israel J. Math. 19 63-74 (1974)

[P3] Procesi, C.: Trace identities and standard diagrams. Ring theory. Lecture Notes in Pure and Appl. Mathematics 51, M. Dekker, 1979, pp. 191-218

[P4] Procesi, C.: A formal inverse to the Cayley Hamilton theorem. J. Algebra 107 63-74 (1987)

[R] Rosso, M.: Analogues de la forme de Killing et du théorème d'Harish-Chandra pour les groupes quantiques. Ann. Sci. Ec. Norm. Sup. 23, 445-467 (1990)

[Ta] Tanisaki, T.: Killing forms, Harish-Chandra isomorphisms, an universal $R$-matrices for quantum algebras. RIMS-816 preprint (1991)

[T] Tits, J.: Sur les constants de structure et le théorème d'existence des algebres de Lie semi-simple. Publ. Math. IHES 31, 21-58 (1966)

[W-K] Weisfeiler, B.Ju.; Kac, V.G.: On irreducible representations of the Lie $p$-algebras. Funct. Anal. Appl. 5, 28-36 (1971)

Communicated by A. Jaffe 
